\title{
LIMITS OF A DISTRIBUTION FUNCTION DETERMINED BY ABSOLUTE MOMENTS AND INEQUALITIES SATISFIED BY ABSOLUTE MOMENTS*
}

\author{
BY

\section{ABRAHAM WALD $\dagger$}

1. Introduction. Denote by $X$ a chance variable and by $P(\alpha<X<\beta)$ the probability that $\alpha<X<\beta$. Similarly denote by $P(X<\beta)$ the probability that $X<\beta$ and by $P(X=\beta)$ the probability that $X=\beta$. For any positive integer $r$ the expected value $E\left|X-x_{0}\right|^{r}$ of $\left|X-x_{0}\right|^{r}$ is called the absolute moment of order $r$ about $x_{0}$, where $x_{0}$ denotes a certain real value. If the absolute moments $M_{i_{1}}=E\left|X-x_{0}\right| i_{1}, \cdots, M_{i_{j}}=E\left|X-x_{0}\right| i_{i}$ of a chance variable $X$ are given (and no further data about $X$ are known), then we shall say for any positive number $d$ that $a_{d}$ is the sharp lower limit of $P(-d<X$ $\left.-x_{0}<d\right)$ if the following two conditions are fulfilled:

(1) For each chance variable $Y$ for which $E\left|Y-x_{0}\right|^{i_{\nu}}=E\left|X-x_{0}\right|^{i_{\nu}}$ $(\nu=1, \cdots, j)$ the inequality $P\left(-d<Y-x_{0}<d\right) \geqq a_{d}$ holds.

(2) To each $\epsilon>0$ a chance variable $Y$ can be given such that $E\left|Y-x_{0}\right|^{i_{\nu}}$ $=\left.E\left|X-x_{0}\right|\right|^{i_{\nu}}(\nu=1, \cdots, j)$ and $P\left(-d<Y-x_{0}<d\right)<a_{d}+\epsilon$.

In other words, $a_{d}$ is the greatest lower bound of the probabilities $P\left(-d<Y-x_{0}<d\right)$ formed for all chance variables $Y$ for which the $i_{\nu}$ th absolute moment about $x_{0}$ is equal to the $i_{\nu}$ th absolute moment of $X$ about $x_{0}$ $(\nu=1, \cdots, j)$.

Similarly we shall say that $b_{d}$ is the sharp upper limit of $P\left(-d<X-x_{0}<d\right)$ if $b_{d}$ is the least upper bound of the probabilities $P\left(-d<Y-x_{0}<d\right)$ formed for all chance variables $Y$ for which the $i_{\nu}$ th absolute moment about $x_{0}$ is equal to the $i_{\text {i }}$ th absolute moment of $X$ about $x_{0}(\nu=1, \cdots, j)$.

In this paper we shall give the solution of the following two problems:

PRoBlem 1. The absolute moments of the order $i_{1}, \cdots, i_{j}$ of a chance variable $X$ are given about the point $x_{0}$, where $i_{1}, \cdots, i_{j}$ denote any positive integers. It is required to determine the sharp lower and sharp upper limit of $P\left(-d<X-x_{0}<d\right)$ for any positive value $d$.

Problem 2. A real value $x_{0}$ and a system of $j$ positive integers $i_{1}, \cdots, i_{j}$ are given. What are the necessary and sufficient conditions which must be satisfied

* Presented to the Society, February 25, 1939; received by the editors February 15, 1939.

$\dagger$ This research was done under a grant-in-aid from the Carnegie Corporation of New York. 
by $j$ positive numbers $a_{1}, \cdots, a_{j}$ that a chance variable $X$ exists for which the $i_{\nu}$ th moment about $x_{0}$ is equal to $a_{\nu}(\nu=1, \cdots, j)$ ?

The solution of Problem 1 is a generalization of the inequality of Markoff. In fact, the inequality of Markoff can be written as follows:

$$
P\left(-d<X-x_{0}<d\right) \geqq 1-M_{r} / d^{r},
$$

where $d$ denotes an arbitrary positive value and $M_{r}$ denotes the $r$ th absolute moment of $X$ about $x_{0}$. As is well known, the inequality (1) cannot be improved for $d \geqq M_{r}^{1 / r}$, that is to say that $1-M_{r} / d^{r}$ is the sharp lower limit of $P\left(-d<X-x_{0}<d\right)$ for $d \geqq M_{r}{ }^{1 / r}$. The generalization in our Problem 1 consists in the circumstance that instead of a single moment $M_{r}$ we consider a finite number of moments $M_{i_{1}}, \cdots, M_{i_{j}}$, and besides the sharp lower limit of $P\left(-d<X-x_{0}<d\right)$ also its sharp upper limit is to be determined. The inequality (1) is called for $r=2$ also the inequality of Tshebysheff.

Some results concerning the case when two moments $M_{r}$ and $M_{s}$ are given, have been obtained by different authors. A. Guldberg* gave the following formula:

$$
P\left(\left|X-x_{0}\right|<\lambda M_{r}^{1 / r}\right) \geqq 1-\frac{1}{\lambda^{8}}\left(\frac{M_{s}^{1 / s}}{M_{r}^{1 / r}}\right)^{s} .
$$

If we substitute $2 k$ for $s$, and 2 for $r$, we get the inequality of $\mathrm{K}$. Pearson. $\dagger$ By other substitutions we get the formula of E. Lurquin. $\ddagger$ It is easy to show that the limit given in (2) is not sharp.

P. Cantelli§ gave a formula in case that $s=2 r$. His formula can be written as follows:

(3a) If $M_{r} / d^{r} \leqq M_{2 r} / d^{2 r}$, then $P\left(\left|X-x_{0}\right|<d\right) \geqq 1-M_{r} / d^{r}$.

(3b) If $M_{r} / d^{r}>M_{2 r} / d^{2 r}$, then

$$
P\left(\left|X-x_{0}\right|<d\right) \geqq 1-\frac{M_{2 r}-M_{r}^{2}}{\left(d^{r}-M_{r}\right)^{2}+M_{2 r}-M_{r}^{2}} .
$$

The writer of this article gave in a previous paper $\|$ some results concerning the general case and the sharp lower limit of $P\left(-d<X-x_{0}<d\right)$ if two

* A. Guldberg, Comptes Rendus de l'Académie des Sciences, Paris, vol. 175, p. 679.

$\dagger$ K. Pearson, Biometrika, vol. 12 (1918-1919).

$\ddagger$ E. Lurquin, Comptes Rendus de l'Académie des Sciences, Paris, vol. 175, p. 681.

$\S$ Cantelli's formula and its demonstration are given in the book of M. Fréchet, Recherches Théoriques Modernes sur la Théorie des Probabilités, Paris, 1937, pp. 123-126.

\| A. Wald, A generalization of Markoff's inequality, Annals of Mathematical Statistics, December, 1938. 
moments $M_{r}$ and $M_{s}$ are given, where $r$ and $s$ denote arbitrary positive integers. If $s=2 r$ the formula reduces to Cantelli's formula.

In case of consecutive algebraic moments, that is to say, if $M_{1}, \cdots, M_{j}$ are given and $M_{i}=E\left(X-x_{0}\right)^{i}(i=1, \cdots, j)$, Tshebysheff determined the sharp lower and sharp upper limit of the distribution function $P(X<d)$. These inequalities are called Tshebysheff's inequalities. The first proof of these inequalities was given by Markoff in 1884 and the same proof was discovered almost at the same time by Stieltjes.*

The solution of Problem 2 is well known $\dagger$ if $i_{1}, \cdots, i_{j}$ are consecutive integers, that is to say, if $i_{\nu}=\nu(\nu=1, \cdots, j)$ and if $a_{\nu}(\nu=1, \cdots, j)$ is the $\nu$ th algebraic moment, that is to say, $a_{v}=E\left(X-x_{0}\right)^{\nu}$. In this paper we shall give the solution for absolute moments and for arbitrary positive integers $i_{1}, \cdots, i_{j}$.

2. Reduction of the problem to the case of nonnegative chance variables. We shall call a chance variable $X$ nonnegative if $P(X<0)=0$. Since the moments of the nonnegative chance variable $Y=\left|X-x_{0}\right|$ about the origin are equal to the absolute moments of $X$ about $x_{0}$ and since

$$
P(Y<d)=P\left(-d<X-x_{0}<d\right),
$$

the following proposition holds true:

Proposition 1. Denote by $M_{i_{1}}, \cdots, M_{i_{j}}$ the absolute moments of order $i_{1}, \cdots, i_{j}$ of a certain chance variable $X$ about the point $x_{0}$. There exists a nonnegative chance variable $Y$ such that the $i_{\text {v }}$ th moment of $Y$ about the origin is equal to $M_{i_{\nu}}(\nu=1, \cdots, j)$. The greatest lower (least upper) bound of the probabilities $P\left(-d<Z-x_{0}<d\right)$ is equal to the greatest lower (least upper) bound of the probabilities $P\left(Z^{\prime}<d\right)$, where $P\left(-d<Z-x_{0}<d\right)$ is formed for all chance variables $Z$ for which the $i_{\nu}$ th absolute moment about $x_{0}$ is equal to $M_{i_{\nu}}$ and $P\left(Z^{\prime}<d\right)$ is formed for all nonnegative chance variables $Z^{\prime}$ for which the $i_{\nu}$ th moment about the origin is equal to $M_{i_{\nu}}(\nu=1, \cdots, j)$.

On account of Proposition 1 we can restrict ourselves to the consideration of nonnegative chance variables and of the moments about the origin. Throughout the following developments we shall understand by a chance variable a nonnegative chance variable and by moments the moments about the origin.

3. Some definitions and propositions. Let us begin with some definitions.

* See, for instance, J. Uspensky, Introduction to Mathematical Probability, New York, McGrawHill, 1937, pp. 373-380.

† See, for instance, R. von Mises, Wahrscheinlichkeitsrechnung und ihre Anwendung in der Statistik und theoretischen Physik, Deuticke, Leipzig, 1931, pp. 247-248. 
Definition 1. A chance variable $X$ is said to be an arithmetic chance variable, if there exist a finite system of different numbers $x_{1}, \cdots, x_{k}$ such that $\sum_{i=1}^{k} P\left(X=x_{i}\right)=1$.

Definition 2. A chance variable $X$ for which $k$ different positive values $x_{1}, \cdots, x_{k}$ exist such that $P\left(X=x_{i}\right)>0(i=1, \cdots, k)$ and $\sum_{i=1}^{k} P\left(X=x_{i}\right)=1$, is called an arithmetic chance variable of the degree $k$.

Definition 3. A chance variable $X$ is said to be an arithmetic chance variable of the degree $k+1 / 2$ if $P(X=0)>0$ and if there exist $k$ different positive values $x_{1}, \cdots, x_{k}$ such that $P\left(X=x_{i}\right)>0(i=1, \cdots, k)$ and $\sum_{i=1}^{k} P\left(X=x_{i}\right)+P(X=0)$ $=1$.

Definition 4. Denote by $M_{i_{1}}, \cdots, M_{i_{j}}$ the moments of the order $i_{1}, \cdots, i_{j}$ of a certain chance variable $X$. A chance variable $Y$ is said to be characteristic relative to $M_{i_{1}}, \cdots, M_{i_{j}}$ if the $i_{\nu}$ th moment of $Y$ is equal to $M_{i_{\nu}}(\nu=1, \cdots, j)$ and $Y$ is an arithmetic chance variable of the degree less than or equal to $(j+1) / 2$. $A$ characteristic chance variable is said to be degenerate if its degree is less than $(j+1) / 2$.

Definition 5. We shall say that the numbers $M_{i_{1}}, \cdots, M_{i_{j}}$ can be realized as moments of the order $i_{1}, \cdots, i_{j}$ if there exists a chance variable $X$ such that the $i_{\nu}$ th moment of $X$ is equal to $M_{i_{\nu}}(\nu=1, \cdots, j)$.

Definition 6. A function $f(x)$ defined for all real values $x$ is said to change its sign at the point $x=\alpha$ if the following conditions are fulfilled:

(1) If $f(x)=0$ for all values $x<\alpha$, then any open interval containing $\alpha$ must contain at least one value $\alpha^{\prime}$ such that $f(\alpha) f\left(\alpha^{\prime}\right)<0$.

(2) If $f(x)$ is not identically zero for $x<\alpha$, then any open interval which contains $\alpha$ and a point $\beta<\alpha$ for which $f(\beta) \neq 0$, must also contain two points $\alpha_{1}$ and $\alpha_{2}$ such that $\alpha_{1} \leqq \alpha, \alpha_{2} \geqq \alpha$ and $f\left(\alpha_{1}\right) f\left(\alpha_{2}\right)<0$.

By the number of changes in sign of $f(x)$ we shall understand the number of points at which $f(x)$ changes its sign. Similarly we shall understand by the number of changes in sign in an (open or closed) interval $A$, the number of points of $A$ at which $f(x)$ changes its sign.

It is easy to prove that if $f\left(\alpha_{1}\right) f\left(\alpha_{2}\right)<0$ then there exists at least one point of the closed interval $\left[\alpha_{1}, \alpha_{2}\right]$ at which $f(x)$ changes its sign. In order to prove this, let us assume that $\alpha_{1}<\alpha_{2}$ and denote by $\alpha$ the greatest lower bound of all values $\gamma$ of the interval $\left[\alpha_{1}, \alpha_{2}\right]$ for which $f\left(\alpha_{1}\right) f(\gamma)<0$. It is obvious that $\alpha_{1} \leqq \alpha \leqq \alpha_{2}$. We shall show that $f(x)$ changes its sign at $\alpha$. If $\alpha=\alpha_{1}$ then from the definition of $\alpha$ it follows that any open interval containing $\alpha$ contains also a point $\alpha^{\prime}$ such that

$$
f\left(\alpha_{1}\right) f\left(\alpha^{\prime}\right)=f(\alpha) f\left(\alpha^{\prime}\right)<0 .
$$


Hence $f(x)$ changes its sign at $\alpha$. If $\alpha>\alpha_{1}$ then for any value $\delta \geqq \alpha_{1}$ and less than $\alpha, f(\delta)$ has the same sign as $f\left(\alpha_{1}\right)$ or is equal to zero. From this fact it follows easily that any open interval which contains $\alpha$ and a value $\beta<\alpha$ for which $f(\beta) \neq 0$, contains also two points $\beta_{1}$ and $\beta_{2}$ such that $\beta_{1} \leqq \alpha, \beta_{2} \geqq \alpha$ and $f\left(\beta_{1}\right) f\left(\beta_{2}\right)<0$. Hence $f(x)$ changes its sign at $\alpha$ in any case.

If $f(x)$ does not change its sign at any point of the (open or closed) interval $I$, then $f(\alpha) f(\beta) \geqq 0$ for any two points $\alpha, \beta$ of $I$. In fact if $I$ should contain two points $\alpha, \beta$ such that $f(\alpha) f(\beta)<0$, then $[\alpha, \beta]$ and therefore also $I$ must contain a point $\gamma$ at which $f(x)$ changes its sign, in contradiction to our assumption.

We shall prove now the

Proposition 2. If $X$ denotes an arithmetic chance variable of degree $k$ and $Y$ denotes an arbitrary chance variable, then the number of changes in sign of $D(x)=P(X<x)-P(Y<x)$ is less than or equal to $2 k-1$.

Let us first consider the case that $k$ is integral. In this case there are $k$ different positive values $\alpha_{1}, \cdots, \alpha_{k}$ such that $P\left(X=\alpha_{i}\right)>0(i=1, \cdots, k)$ and $\sum_{i=1}^{k} P\left(X=\alpha_{i}\right)=1$. It is obvious that at most one change in sign of $D(x)$ can take place in the interior of the interval $I_{i}=\left[\alpha_{i}, \alpha_{i+1}\right](i=1, \cdots, k-1)$. Besides the changes in sign in the interior of the intervals $I_{1}, \cdots, I_{k-1}$ a change in sign can only occur at the points $\alpha_{1}, \cdots, \alpha_{k}$. Hence the total number of changes in sign cannot exceed $(k-1)+k=2 k-1$.

If $k=k^{\prime}+1 / 2$, where $k^{\prime}$ denotes a nonnegative integer, then $P(X=0)>0$ and there exist $k^{\prime}$ different positive values $\alpha_{1}, \cdots, \alpha_{k^{\prime}}$ such that $P\left(X=\alpha_{i}\right)>0$ $\left(i=1, \cdots, k^{\prime}\right)$ and $\sum_{i=1}^{k^{\prime}} P\left(X=\alpha_{i}\right)+P(X=0)=1$. Let us denote the point 0 by $\alpha_{0}$. It is obvious that in the interior of the interval $I_{i}=\left[\alpha_{i}, \alpha_{i+1}\right]$ $\left(i=0,1, \cdots, k^{\prime}-1\right)$ at most one change in sign of $D(x)$ can take place. Further changes in sign ran occur only at the points $\alpha_{1}, \cdots, \alpha_{k^{\prime}}$. Hence the total number of changes in sign cannot exceed $2 k^{\prime}=2 k-1$.

Proposition 3. If $X$ and $Y$ denote two arithmetic chance variables of degree less than or equal to $k$, then the number of changes in sign of $D(x)$ $=P(X<x)-P(Y<x)$ is less than or equal to $2 k-2$.

First let us consider the case that both chance variables $X$ and $Y$ are of the degree $k$. If $k$ is a positive integer, then there exist two systems of $k$ positive values $\alpha_{1}, \cdots, \alpha_{k}$ and $\beta_{1}, \cdots, \beta_{k}$ such that

$$
\sum_{i=1}^{k} P\left(X=\alpha_{i}\right)=\sum_{i=1}^{k} P\left(Y=\beta_{i}\right)=1 .
$$

We may assume that $\alpha_{1} \leqq \beta_{1}$. (If it happens that $\beta_{1}<\alpha_{1}$, we can change the notation.) Hence $D(x)$ has no change in sign at the point $\alpha_{1}$. Since in the in- 
terior of the interval $\left[\alpha_{i}, \alpha_{i+1}\right](i=1, \cdots, k-1)$ at most one change in sign can take place and further changes in sign can occur only at the points $\alpha_{2}, \alpha_{3}, \cdots, \alpha_{k}$, the total number of changes in sign cannot exceed $(k-1)$ $+(k-1)=2 k-2$. If $k=k^{\prime}+1 / 2$, where $k^{\prime}$ denotes a nonnegative integer, then $P(X=0)>0, P(Y=0)>0$ and there exist two systems of $k^{\prime}$ positive numbers $\alpha_{1}, \cdots, \alpha_{k^{\prime}} ; \beta_{1}, \cdots, \beta_{k^{\prime}}$ such that $P\left(X=\alpha_{i}\right)>0, P\left(Y=\beta_{i}\right)>0\left(i=1, \cdots, k^{\prime}\right)$ and

$$
\sum_{i=1}^{k^{\prime}} P\left(X=\alpha_{i}\right)+P(X=0)=\sum_{i=1}^{k^{\prime}} P\left(Y=\beta_{i}\right)+P(Y=0)=1 .
$$

We may assume that $\alpha_{1} \leqq \beta_{1}$. It is obvious that $D(x)$ has no change in sign in the interior of the interval $\left[0, \alpha_{1}\right]$. Since $D(x)$ has at most one change in sign in the interior of the interval $\left[\alpha_{i}, \alpha_{i+1}\right]\left(i=1, \cdots, k^{\prime}-1\right)$, and since further changes in sign can occur only at the points $\alpha_{1}, \cdots, \alpha_{k^{\prime}}$, the total number of changes in sign cannot exceed $2 k^{\prime}-1=2 k-2$. Hence Proposition 3 is proved if $X$ and $Y$ are of the degree $k$.

Let us now consider the case that $X$ or $Y$ or both are of degree less than $k$. Let for instance the degree of $X$ be less than $k$. Hence the degree of $X$ is less than or equal to $k-1 / 2$ and therefore on account of Proposition 2 the number of changes in sign of $D(x)$ cannot exceed $2(k-1 / 2)-1=2 k-2$.

Proposition 4. If $X$ and $Y$ denote two arithmetic chance variables of degree less than or equal to $k>1$ and if there exists a positive number $\alpha$ such that $P(X=\alpha)>0$ and $P(Y=\alpha)>0$, then the number of changes in sign of $D(x)$ $=P(X<x)-P(Y<x)$ is less than or equal to $2 k-3$.

We may assume that $P(X<\alpha) \leqq P(Y<\alpha)$. Consider first the case that $P(Y<\alpha)>0$ and denote by $\alpha^{\prime}$ the greatest value less than $\alpha$ for which $P\left(Y=\alpha^{\prime}\right)>0$. It is obvious that $D(x)$ has no change in sign in the interior of the interval $\left[\alpha^{\prime}, \alpha\right]$. If $D(x)$ is identically zero in the interior of $\left[\alpha^{\prime}, \alpha\right]$, then $D(x)$ has no change in sign at $\alpha^{\prime}$. If $D(x)$ is not identically zero in the interior of $\left[\alpha^{\prime}, \alpha\right]$ and if $P(X \leqq \alpha) \leqq P(Y \leqq \alpha)$, then $D(x)$ has no change in sign at $\alpha$. Finally if $P(X \leqq \alpha)>P(Y \leqq \alpha)$ and $\alpha^{\prime \prime}$ denotes the smallest value greater than $\alpha$ for which $P\left(Y=\alpha^{\prime \prime}\right)>0$, then $D(x)$ has no change in sign in the interior of the interval $\left[\alpha, \alpha^{\prime \prime}\right]$. Hence in any case the number of changes in sign of $D(x)$ cannot exceed $(2 k-1)-2=2 k-3$. Now we have to prove Proposition 4 if $P(Y<\alpha)=0$. Since $P(X<\alpha) \leqq P(Y<\alpha)=0, D(x)$ has no change in sign at $\alpha$. If $P(X \leqq \alpha)=P(Y \leqq \alpha)=1$, then $D(x)$ has no change in sign at all and Proposition 4 is proved. We have to consider only the case that at least one of the values $P(X \leqq \alpha), P(Y \leqq \alpha)$ is less than 1 . Let us assume that $P(X \leqq \alpha) \geqq P(Y \leqq \alpha)$. The probability $P(Y \leqq \alpha)$ must be less than 1 , 
since otherwise also $P(X \leqq \alpha)$ would be equal to 1 , in contradiction to our assumption. Denote by $\beta$ the smallest value greater than $\alpha$ for which $P(Y=\beta)$ $>0$. Then $D(x)$ has obviously no change in sign in the interior of $[\alpha, \beta]$ and therefore the total number of changes in sign cannot exceed $2 k-3$. If $P(X \leqq \alpha)<P(Y \leqq \alpha)$, then denote by $\beta$ the smallest value greater than $\alpha$ for which $P(X=\beta)>0$. The function $D(x)$ has no change in sign in the interior of $[\alpha, \beta]$ and therefore also in this case the total number of changes in sign of $D(x)$ cannot exceed $2 k-3$.

Proposition 5. Denote by $X$ and $Y$ two chance variables. If the $i_{v}$ th moment $(\nu=1, \cdots, j)$ of $X$ is finite and equal to the $i_{\nu}$ th moment of $Y$, then $D(x)$ $=P(X<x)-P(Y<x)$ must have at least $j$ changes in sign, unless $D(x)$ is identically zero.

Denote $P(X<x)$ by $V_{1}(x)$ and $P(Y<x)$ by $V_{2}(x)$. Since the $i_{\nu}$ th moment of $X$ is equal to the $i_{\nu}$ th moment of $Y(\nu=1, \cdots, j)$, the Stieltjes integral

$$
I=\int_{0}^{\infty}\left(a_{1} x^{i_{1}}+\cdots+a_{j} x^{i_{i}}\right) d\left[V_{1}(x)-V_{2}(x)\right]=0
$$

for arbitrary real values $a_{1}, \cdots, a_{j}$. Denote the integral

$$
\int_{0}^{\lambda}\left(a_{1} x^{i_{1}}+\cdots+a_{j} x^{i_{i}}\right) d\left[V_{1}(x)-V_{2}(x)\right]
$$

by $I_{\lambda}$. It is obvious that

$$
\lim _{\lambda=\infty} I_{\lambda}=I=0 .
$$

We get by integration by parts

$$
\begin{aligned}
I_{\lambda}= & \left(a_{1} \lambda^{i_{1}}+\cdots+a_{j} \lambda^{i_{j}}\right)\left[V_{1}(\lambda)-V_{2}(\lambda)\right] \\
& -\int_{0}^{\lambda}\left(i_{1} a_{1} x^{i_{1}-1}+\cdots+i_{j} a_{j} x^{i_{j}-1}\right)\left[V_{1}(x)-V_{2}(x)\right] d x .
\end{aligned}
$$

Now we shall show that

$$
\lim _{\lambda=\infty} \lambda^{i \nu}\left[V_{1}(\lambda)-V_{2}(\lambda)\right]=0, \quad \nu=1, \cdots, j .
$$

Since

$$
\lambda^{i_{\nu}}\left[V_{1}(\lambda)-V_{2}(\lambda)\right]=\lambda^{i_{\nu}}\left[1-V_{2}(\lambda)\right]-\lambda^{i_{\nu}}\left[1-V_{1}(\lambda)\right]
$$

we have only to show that

$$
\lim \lambda^{i_{\nu}}\left[1-V_{r}(\lambda)\right]=0, \quad r=1,2 .
$$

It is obvious that for any $\lambda>0$ 


$$
\lambda^{i_{\nu}}\left[1-V_{r}(\lambda)\right]=\int_{\lambda}^{\infty} \lambda^{i_{\nu}} d V_{r}(x) \leqq \int_{\lambda}^{\infty} x^{i_{\nu}} d V_{r}(x) .
$$

Since the $i_{\nu}$ th moments of $X$ and $Y$ are finite, we have

$$
\lim _{\lambda=\infty} \int_{\lambda}^{\infty} x^{i} d V_{r}(x)=0,
$$

and therefore (8) and (7) must hold. Then we get from the equations (4), (5) and (6)

$$
\int_{0}^{\infty}\left(i_{1} a_{1} x^{i_{1}-1}+\cdots+i_{j} a_{j} x^{i_{j}-1}\right)\left[V_{1}(x)-V_{2}(x)\right] d x=0 .
$$

Let us suppose that the number of changes in sign of $D(x)=V_{1}(x)-V_{2}(x)$ is less than $j$ and denote by $\alpha_{1}<\alpha_{2}<\cdots<\alpha_{k}(k<j)$ the points at which $D(x)$ changes its sign. It is obvious that $\alpha_{1}>0$. Consider the intervals

$$
I_{1}=\left[0, \alpha_{1}\right], I_{2}=\left[\alpha_{1}, \alpha_{2}\right], \cdots, I_{k}=\left[\alpha_{k-1}, \alpha_{k}\right], I_{k+1}=\left[\alpha_{k}, \infty\right] .
$$

$D(x)$ is in the interior of the interval $I_{\nu}$ either everywhere nonnegative or everywhere nonpositive, and if $D(x) \geqq 0(\leqq 0)$ in the interior of $I_{\nu}$ then $D(x) \leqq 0 \quad(\geqq 0)$ in the interior of $I_{\nu-1}(\nu=2,3, \cdots, k+1)$. We put $a_{k+2}=a_{k+3}=\cdots=a_{i}=0$ and consider the $k$ equations

$$
\begin{aligned}
& i_{1} a_{1} \alpha_{1}^{i_{1}-1}+\cdots+i_{k+1} a_{k+1} \alpha_{1}^{i_{k+1}-1}=0, \\
& \text {. . . . . . . . . . }, \\
& i_{1} a_{1} \alpha_{k}^{i_{1}-1}+\cdots+i_{k+1} a_{k+1} \alpha_{k}^{i_{k+1}-1}=0 .
\end{aligned}
$$

There exists a system of roots $a_{1}=a_{1}{ }^{\prime}, \cdots, a_{k+1}=a_{k+1}{ }^{\prime}$ such that at least one among them is not equal to zero. Denote the polynomial

$$
i_{1} a_{1}^{\prime} x^{i_{1}-1}+\cdots+i_{k+1} a_{k+1}^{\prime} x^{i_{k+1}-1}
$$

by $Q(x)$. It is obvious that $\alpha_{1}, \cdots, \alpha_{k}$ are roots of $Q(x)$. Since the number of changes in sign in the sequence of the coefficients of $Q(x)$ is less than or equal to $k, Q(x)$ has at most $k$ positive roots. Hence $\alpha_{1}, \cdots, \alpha_{k}$ must be simple roots of $Q(x)$ and therefore the sign of $Q(x)$ in the interval $I_{\nu}=\left[\alpha_{\nu-1}, \alpha_{\nu}\right]$ is opposite to the sign of $Q(x)$ in the interval $I_{\nu-1}(\nu=2, \cdots, k+1)$. From this fact it follows that the product $Q(x)\left[V_{1}(x)-V_{2}(x)\right]$ has no change of sign at all. Hence the integral

$$
\int_{0}^{\infty} Q(x)\left[V_{1}(x)-V_{2}(x)\right] d x
$$

can vanish only if $V_{1}(x)-V_{2}(x)$ is identically zero. This proves Proposition 5. 
From Propositions 2 and 5 follows easily

Proposition 6. If $X$ denotes an arithmetic chance variable of degree $k$ and $Y$ denotes a chance variable such that $2 k$ different moments of $Y$ are equal to the corresponding moments of $X$, then $P(Y<x)$ is identically equal to $P(X<x)$.

From Propositions 3 and 5 we get the

Proposition 7. For each system of moments $M_{i_{1}}, \cdots, M_{i_{j}}$ there exists at most one chance variable which is characteristic relative to $M_{i_{1}}, \cdots, M_{i_{j}}$.

We shall now prove the

Proposition 8. If the chance variable $X$ is characteristic relative to $M_{i_{1}}, \cdots, M_{i_{j}}$, and $M_{r}^{\prime}$ is the rth moment of $X$, where $r>i_{1}, \cdots, i_{j}$, then $M_{i_{1}}, \cdots, M_{i_{j}}, M_{r}$ for $M_{r}<M_{r}^{\prime}$ cannot be realized as moments of the orders $i_{1}, \cdots, i_{j}, r$.

Let us suppose that there exists a chance variable $Y$ with the moments $M_{i_{1}}, \cdots, M_{i_{j}}, M_{r}$ where $M_{r}<M_{r}^{\prime}$. We shall deduce a contradiction from this assumption. We can assume that $Y$ is an arithmetic chance variable, because according to a well known theorem a finite system of moments can always be realized by an arithmetic chance variable. On account of Proposition $5, D(x)=P(Y<x)-P(X<x)$ must have at least $j$ changes in sign. Since $X$ is a characteristic chance variable, the number of changes in sign of $D(x)$ cannot exceed $j$; hence the number of changes in sign must be equal to $j$. It is easy to see that the number of changes in sign can be equal to $j$ only if the greatest value $x^{\prime}$ for which $P\left(Y=x^{\prime}\right)>0$ is greater than the greatest value $x^{\prime \prime}$ for which $P\left(X=x^{\prime \prime}\right)>0$. We denote by $Y_{d}$ the arithmetic chance variable defined as follows:

$$
\begin{array}{lr}
P\left(Y_{d}=d\right)=\frac{M_{r}^{\prime}-M_{r}}{d^{r}}, & P\left(Y_{d}=x^{\prime}\right)=P\left(Y=x^{\prime}\right)-\frac{M_{r}^{\prime}-M_{r},}{d^{r}}, \\
P\left(Y_{d}=x\right)=P(Y=x), & \text { for } x \neq d, x^{\prime},
\end{array}
$$

where $d>x^{\prime}$ and $P\left(Y=x^{\prime}\right)>\left(M_{r}^{\prime}-M_{r}\right) / d^{r}$. The differences between the moments (of the orders $\left.i_{1}, \cdots, i_{j}, r\right)$ of $X$ and the corresponding moments of $Y_{d}$ become arbitrarily small if we choose $d$ sufficiently large. It is obvious that $P(X<x)-P\left(Y_{d}<x\right)$ has always the same sign as $P(X<x)-P(Y<x)$. Since the number of changes in sign of $D(x)$ is equal to $j$, a polynomial $P(x)=a_{1} x^{i_{1}}+\cdots+a_{j} x^{i_{j}}+a_{r} x^{r}$ can be given such that $P^{\prime}(x)=i_{1} a_{1} x^{i_{1}-1}+\cdots$ $+i_{j} a_{j} x^{i_{j}-1}+r a_{r} x^{r-1}$ has always the same sign as that of $P(X<x)-P(Y<x)$ and therefore has also the same sign as that of $P(X<x)-P\left(Y_{d}<x\right)$ for any $d$. Since $P\left(Y_{d}<x\right)=P(Y<x)$ for any $x<x^{\prime}$ and since $P(Y<x)-P(X<x)$ is not equal to zero for any $x<x^{\prime}$, the integral 


$$
\int_{0}^{\infty} P^{\prime}(x)\left[P\left(Y_{d}<x\right)-P(X<x)\right] d x
$$

cannot converge towards zero if $d \rightarrow \infty$. But on the other hand the moments of the order $i_{1}, \cdots, i_{j}, r$ of $Y_{d}$ converge towards the corresponding moments of $X$ if $d \rightarrow \infty$ and therefore, as can easily be shown, the above integral must converge towards zero. Hence we get a contradiction and our proposition is proved.

Definition 7. A sequence $\left\{X_{i}\right\}$ of chance variables is said to be convergent towards the chance variable $X$, in symbols $\lim _{i=\infty} X_{i}=X$, if $\left\{P\left(X_{i}<x\right)\right\}$ $(i=1,2, \cdots$, ad inf.) converges uniformly towards $P(X<x)$ in any closed set of values of $x$ which does not contain any point of discontinuity of $P(X<x)$.

In the following development we shall understand by " $X$ is equal to $Y$," in symbols $X=Y$, that $P(X<x)$ is identically equal to $P(Y<x)$.

For any integer $r$ we shall denote the $r$ th moment of a chance variable $X$ also by $M_{r}(X)$.

Definition 8. A chance variable $X_{\alpha}$ defined for any point $\alpha$ of a domain $D$ is said to be a continuous function of $\alpha$ in $D$, if for any $\alpha$ in $D$ and for any sequence of points $\left\{\alpha_{i}\right\}$ in $D$ which converges towards $\alpha, \lim _{i=\infty} X_{\alpha_{i}}=X_{\alpha}$.

Now we shall prove

Proposition 9. If $\left\{X_{i}\right\}(i=1,2, \cdots$, ad inf. $)$ denotes a sequence of arithmetic chance variables of degree less than a certain integer $n$ which converges towards the chance variable $X$, and if for a certain positive integer $r,\left\{M_{r}\left(X_{i}\right)\right\}$ $(i=1,2, \cdots$, ad inf. $)$ is bounded, then $\lim _{i=\infty} M_{s}\left(X_{i}\right)=M_{s}(X)$ for any positive integer $s<r$.

It is obvious that $X$ is an arithmetic chance variable of degree less than $n$. Denote by $\epsilon_{s}\left(X_{i}, t\right)$ the Stieltjes integral $\int_{t}^{\infty} x^{s} d P\left(X_{i}<x\right)$ where $t>0$. It is obvious that for any positive value $t$ for which $P(X \geqq t)=0$

$$
\lim _{i=\infty}\left[M_{k}\left(X_{i}\right)-\epsilon_{k}\left(X_{i}, t\right)\right]=M_{k}(X), \quad k=1,2, \cdots, \text { ad inf. }
$$

Suppose that $\left\{M_{r}\left(X_{i}\right)\right\}$ is bounded for a certain $r$. Since $\epsilon_{r}\left(X_{i}, t\right) \leqq M_{r}\left(X_{i}\right)$ $\left(i=1, \cdots\right.$, ad inf.), $\left\{\epsilon_{r}\left(X_{i}, t\right)\right\}$ must also be bounded. That is to say, there exists a positive value $N$ such that $\epsilon_{r}\left(X_{i}, t\right)<N$ for any integer $i$ and for any positive value $t$. Hence $\epsilon_{s}\left(X_{i}, t\right)<N / t$ for $s=1,2, \cdots, r-1$. Let us now suppose that for a certain $s<r, M_{s}\left(X_{i}\right)$ does not converge towards $M_{s}(X)$. Then a subsequence $\left\{X_{i_{j}}\right\}\left(j=1, \cdots\right.$, ad inf.) can be given such that $M_{s}\left(X_{i_{j}}\right)$ converges with increasing $j$ towards a value $M_{s}^{\prime} \neq M_{s}(X)$. We choose a value $t$ for which $P(X<t)=1$ and $N / t<\left|M_{s}^{\prime}-M_{s}(X)\right| / 2$. It is obvious that for 
this $t, M_{s}\left(X_{i}\right)-\epsilon_{s}\left(X_{i}, t\right)$ cannot converge towards $M_{s}(X)$. Hence we have a contradiction and the assumption that $M_{s}\left(X_{i}\right)$ does not converge towards $M_{s}(X)$ is proved to be an absurdity.

Proposition 10. If $\left\{X_{i}\right\}$ ( $i=1,2, \cdots$, ad inf.) denotes a sequence of arithmetic chance variables of degree less than or equal to $k$, and if there exists an integer $r$ for which $\left\{M_{r}\left(X_{i}\right)\right\}(i=1, \cdots$, ad inf.) is bounded, then there exists a subsequence $\left\{X_{i_{j}}\right\}(j=1, \cdots$, ad inf. $)$ which is convergent.

Since $X_{i}$ is of degree less than or equal to $k$, there exists a subsequence $\left\{X_{i}^{\prime}\right\}$ of $\left\{X_{i}\right\}$ such that the number of different values with positive probability is the same for each element of the sequence $\left\{X_{i}^{\prime}\right\}(i=1, \cdots$, ad inf.). Denote this number by $s$. Denote by $\alpha_{i, 1}<\ldots<\alpha_{i, s}$ the values for which $P\left(X_{i}^{\prime}=\alpha_{i, m}\right)>0(m=1, \cdots, s)$. It is obvious that there exists a subsequence $\left\{X_{i_{j}}^{\prime}\right\}(j=1, \cdots$, ad inf. $)$ of the sequence $\left\{X_{i}^{\prime}\right\}$ such that $\lim P\left(X_{i_{j}}^{\prime}=\alpha_{i_{j}, m}\right)$ exists for $m=1, \cdots, s$ and the sequence $\left\{\alpha_{i j, m}\right\} \quad(j=1, \cdots$, ad inf.) converges for each $m \leqq s$ towards a finite value or towards infinity. Since $\left\{M_{r}\left(X_{i}\right)\right\}\left(i=1, \cdots\right.$, ad inf.) is bounded, $\lim _{j=\infty} P\left(X_{i_{j}}^{\prime}=\alpha_{i_{j}, m}\right)=0$ for all $m$ for which $\lim _{j=\infty} \alpha_{i_{j}, m}=\infty$. Since $\alpha_{i, 1}<\alpha_{i, 2}<\cdots<\alpha_{i, s}$, we have $\lim \alpha_{i_{j}, m}=\infty$ if $\lim \alpha_{i_{j}, m-1}=\infty$. Hence there exists an integer $m^{\prime} \leqq s$ such that for any integer $m>m^{\prime}$ and less than or equal to $s, \lim \alpha_{i_{j}, m}=\infty$ and for any integer $m \leqq m^{\prime}, \lim \alpha_{i_{j}, m}$ is finite. Denote $\lim \alpha_{i_{j}, m}$ by $\alpha_{m}$ and $\lim P\left(X_{i_{j}}^{\prime}=\alpha_{i_{j}, m}\right)$ by $p_{m}$ for any $m \leqq m^{\prime}$. It is obvious that $\sum_{m=1}^{m^{\prime}} p_{m}=1$ and $\left\{X_{i_{j}^{\prime}}{ }^{\prime}\right\}$ converges towards the arithmetic chance variable $X$ defined as follows: $P\left(X=\alpha_{m}\right)=p_{m}$ for $m \leqq m^{\prime}$ and $P(X=\alpha)=0$ for any $\alpha \neq \alpha_{1}, \cdots, \alpha_{m^{\prime}}$. Hence Proposition 10 is proved.

Proposition 11. Denote by $\left\{X_{i}\right\}(i=1, \cdots$, ad inf.) a sequence of arithmetic chance variables of degree less than or equal to $k$ for which $\left\{M_{r}\left(X_{i}\right)\right\}$ is bounded for a certain integer $r$. If $\left\{X_{i}\right\}$ does not converge towards the chance variable $X$, then there exists a convergent subsequence $\left\{X_{i_{j}}\right\}$ such that $\lim _{j=\infty} X_{i_{j}}=Y \neq X$.

Since $\left\{X_{i}\right\}$ does not converge towards $X$, there exists a positive $\epsilon$, a sequence of numbers $\left\{\alpha_{i}\right\}$ contained in a closed set which does not contain any discontinuity point of $P(X<x)$, and a subsequence $\left\{X_{i}^{\prime}\right\}$ of $\left\{X_{i}\right\}$ such that $\left|P\left(X_{i}^{\prime}<\alpha_{i}\right)-P\left(X<\alpha_{i}\right)\right|>\epsilon$ for $i=1, \cdots$, ad inf. Hence no subsequence of the sequence $\left\{X_{i}^{\prime}\right\}$ can converge towards $X$. On account of Proposition 10 there exists a convergent subsequence $\left\{X_{i}^{\prime \prime}\right\}$ of the sequence $\left\{X_{i}^{\prime}\right\}$. Hence $\lim X_{i}^{\prime \prime}$ must be different from $X$ and our proposition is proved.

Proposition 12. Denote by $\left\{X_{n}\right\}(n=1, \cdots$, ad inf. $)$ a sequence of arithmetic chance variables of the degree less than or equal to $(j+1) / 2$, where $j$ denotes 
a nonnegative integer. Denote further by $X$ an arithmetic chance variable of degree less than or equal to $(j+1) / 2$ for which $M_{i_{1}}(X), \cdots, M_{i_{j}}(X)$ are finite and $i_{1}<i_{2}<\cdots<i_{j}$ denote certain integers. If $\lim _{n=\infty} M_{i_{\nu}}\left(X_{n}\right)=M_{i_{\nu}}(X)$ $(\nu=1, \cdots, j)$, then $\lim X_{n}=X$.

Let us suppose that $\left\{X_{n}\right\}$ does not converge towards $X$ but $\lim M_{i_{\nu}}\left(X_{n}\right)$ $=M_{i_{\nu}}(X)(\nu=1, \cdots, j)$. According to Proposition 11 there exists a subsequence $\left\{X_{n_{m}}\right\}\left(m=1, \cdots\right.$, ad inf.) such that $\lim _{m=\infty} X_{n_{m}}=X^{\prime} \neq X$. It is obvious that $X^{\prime}$ is of degree less than or equal to $(j+1) / 2$. Consider now the case that there exists an integer $r>i_{j}$ such that $\left\{M_{r}\left(X_{n_{m}}\right)\right\} \quad(m=1, \cdots$, ad inf.) is bounded. Then we have on account of Proposition $9, M_{i_{\nu}}(X)=\lim M_{i_{\nu}}\left(X_{n_{m}}\right)$ $=M_{i_{\nu}}\left(X^{\prime}\right)$. From Proposition 5 it follows that $D(x)=P(X<x)-P\left(X^{\prime}<x\right)$ must have at least $j$ changes in sign. But this is not possible since on account of Proposition 3, $D(x)$ cannot have more than $2(j+1) / 2-2=j-1$ changes in sign. Hence for any integer $r>i_{j},\left\{M_{r}\left(X_{n_{m}}\right)\right\}$ is not bounded. Hence there exists a subsequence $\left\{X_{n_{m}}^{\prime}\right\}$ of $\left\{X_{n_{m}}\right\}$ such that $\lim _{m=\infty} M_{r}\left(X_{n_{m}}^{\prime}\right)=\infty$. Denote by $\alpha_{m}$ the greatest value for which $P\left(X_{n_{m}}^{\prime}=\alpha_{m}\right)>0$. Obviously $\lim \alpha_{m}=\infty$. Since $\lim X_{n_{m}}^{\prime}=X^{\prime}, \lim P\left(X_{n_{m}}^{\prime}=\alpha_{m}\right)$ must be equal to zero. From this fact it follows easily that the degree of $X^{\prime}$ must be less than or equal to $(j+1) / 2-1=(j-1) / 2$. From Proposition 9 we get that

$$
M_{i_{\nu}}\left(X^{\prime}\right)=\lim M_{i_{\nu}}\left(X_{n_{m}}^{\prime}\right)=M_{i_{\nu}}(X), \quad \nu=1, \cdots, j-1 .
$$

Hence according to Proposition 5, $D(x)=P(X<x)-P\left(X^{\prime}<x\right)$ must have at least $j-1$ changes in sign. But this is not possible, because the degree of $X^{\prime}$ is less than or equal to $(j-1) / 2$ and therefore on account of Proposition 2 the number of changes in sign of $D(x)$ is less than or equal to $2(j-1) / 2-1=j-2$. Hence we obtain a contradiction and our assumption that $\left\{X_{n}\right\}$ does not converge towards $X$ is proved to be an absurdity.

Proposition 13. Denote by $M_{i_{1}}, \cdots, M_{i_{j}}$ the moments of the orders $i_{1}<i_{2}<\cdots<i_{i}$ of a certain chance variable $X$. There exists always a chance variable $X^{\prime}$ which is characteristic relative to $M_{i_{1}}, \cdots, M_{i_{j}}$.

We shall prove this proposition by mathematical induction. Proposition 13 is obviously true for $j=1$. We shall suppose that 13 is true for any integer $r \leqq k$. That is to say, we shall make the

Assumption $A_{k}$. Denote by $M_{i_{1}}, \cdots, M_{i_{r}}$ the moments of the orders $i_{1}<\cdots<i_{r}$ of a certain chance variable $X$, where $r \leqq k$. There exists a chance variable $X^{\prime}$ which is characteristic relative to $M_{i_{1}}, \cdots, M_{i_{r}}$.

In order to prove $A_{k+1}$, we shall first prove by means of $A_{k}$ the 
Lemma $B_{k}$. If the chance variable which is characteristic relative to the moments $M_{i_{1}}, \cdots, M_{i_{r}}(r \leqq k)$ is not degenerate, then there exists a positive $\delta$ such that any $r$-tuple $M_{i_{1}}^{\prime}, \cdots, M_{i_{r}}^{\prime}$ can be realized as moments for which

$$
\left|M_{i_{1}}-M_{i_{1}}^{\prime}\right|<\delta, \cdots,\left|M_{i_{r-1}}-M_{i_{r}-1}^{\prime}\right|<\delta
$$

and $M_{i_{r}}^{\prime}>M_{i_{r}}-\delta$.

We shall say that an $n$-tuple $y_{1}, \cdots, y_{n}$ lies in the $\epsilon$-neighborhood of the $n$-tuple $x_{1}, \cdots, x_{n}$ if $\left|x_{1}-y_{1}\right|<\epsilon, \cdots,\left|x_{n}-y_{n}\right|<\epsilon$.

$B_{k}$ is obviously true for $r=1$. We shall prove $B_{k}$ for $r$ by assuming that it is true for $r-1$. Denote by $X$ the chance variable which is characteristic relative to $M_{i_{1}}, \cdots, M_{i_{r}}$ and suppose that $X$ is not degenerate. That is to say, the degree of $X$ is equal to $(r+1) / 2$. According to $A_{k}$ there exists a chance variable $Y$ which is characteristic relative to $M_{i_{1}}, \cdots, M_{i_{r-1}}$. The chance variable $Y$ is also not degenerate. In fact, if $Y$ were degenerate, that is to say, if the degree of $Y$ were less than or equal to $(r-1) / 2$, then according to Proposition 6, $P(X<x)$ would be identically equal to $P(Y<x)$ and therefore also $X$ would be degenerate, in contradiction to our assumption. Hence the degree of $Y$ is equal to $r / 2$. From Propositions 2 and 5 it follows that $M_{i_{r}}(Y) \neq M_{i_{r}}(X)$. Hence on account of Proposition $8, M_{i_{r}}(Y)<M_{i_{r}}(X)$. Since $B_{k}$ is assumed to be true for $r-1$, there exists a positive $\epsilon$ such that any $(r-1)$-tuple $M_{i_{1}}^{\prime}, \cdots, M_{i_{r-1}}^{\prime}$ in the $\epsilon$-neighborhood of $M_{i_{1}}, \cdots, M_{i_{r-1}}$ can be realized as moments. Hence according to $A_{k}$, for each point $M^{\prime}=M_{i_{1}}^{\prime}, \cdots, M_{i_{r-1}}^{\prime}$ of the $\epsilon$-neighborhood of $M=M_{i_{1}}, \cdots, M_{i_{r-1}}$, a chance variable (and only one) exists which is characteristic relative to $M^{\prime}$. Denote by $X\left(M^{\prime}\right)$ the chance variable which is characteristic relative to $M^{\prime}$. From Proposition 12 it follows that $X\left(M^{\prime}\right)$ is a continuous function of $M^{\prime}$ in the $\epsilon$-neighborhood of $M$. For each point $M^{\prime}=M_{i_{1}}^{\prime}, \cdots, M_{i_{r-1}}^{\prime}$ of the $\epsilon$-neighborhood of $M$ the degree of $X\left(M^{\prime}\right)$ must be equal to $r / 2$. In fact, if $X\left(M^{\prime}\right)$ were of degree less than $r / 2$, then $X\left(M^{\prime}\right)$ would be characteristic also relative to $M_{i_{1}}^{\prime}, \cdots, M_{i_{r-2}}^{\prime}$ and therefore on account of Proposition 8 not every point of a neighborhood of $M^{\prime}$ could be realized, in contradiction to the statement that every point of the $\epsilon$-neighborhood of $M$ can be realized. Hence the degree of $X\left(M^{\prime}\right)$ is equal to $r / 2$ for any point $M^{\prime}$ of the $\epsilon$-neighborhood of $M$. From this fact it follows easily that for any integer $n$ the $n$th moment of $X\left(M^{\prime}\right)$ is a continuous function of $M^{\prime}$ in the $\epsilon$-neighborhood of $M$. Since $X(M)=Y$ and $M_{i_{r}}(Y)<M_{i_{r}}(X)$, there exists a positive value $\delta<\epsilon$ such that for any point $M^{\prime}$ of the $\delta$-neighborhood of $M$, the $i_{r}$ th moment of $X\left(M^{\prime}\right)$ is less than $M_{i_{r}}(X)-\delta$. Consider a certain point $M^{\prime}=M_{i_{1}}^{\prime}, \cdots, M_{i_{r-1}}^{\prime}$ of the $\delta$-neighborhood of $M$ and the $(r-1)$-tuple $M_{i_{1}}(d, \eta), \cdots, M_{i_{r-1}}(d, \eta)$ defined as follows: 


$$
M_{i_{1}}(d, \eta)=\frac{M_{i_{1}}^{\prime}-d^{i_{1}} \eta}{1-\eta}, \cdots, M_{i_{r-1}}(d, \eta)=\frac{M_{i_{r-1}}^{\prime}-d^{i_{r-1} \cdot \eta}}{1-\eta}
$$

where $d$ and $\eta$ are positive numbers such that the $(r-1)$-tuple $M_{i_{1}}(d, \eta), \cdots$, $M_{i_{r-1}}(d, \eta)$ is contained in the $\delta$-neighborhood of $M$. Denote by $X(d, \eta)$ the chance variable which is characteristic relative to $M_{i_{1}}(d, \eta), \cdots, M_{i_{r-1}}(d, \eta)$. Denote further by $Y(d, \eta)$ the arithmetic chance variable defined as follows:

$$
\begin{array}{ll}
P[Y(d, \eta)=x]=P[X(d, \eta)=x] \cdot(1-\eta), & \text { for } x \neq d, \\
P[Y(d, \eta)=d]=P[X(d, \eta)=d] \cdot(1-\eta)+\eta . &
\end{array}
$$

It is obvious that the $i_{\nu}$ th moment of $Y(d, \eta)$ is equal to $M_{i}^{\prime}$ $(\nu=1, \cdots, r-1)$. The $i_{r}$ th moment of $Y(d, \eta)$ is a continuous function of $d$ and $\eta$. For $\eta=0, Y(d, \eta)$ is equal to $X\left(M^{\prime}\right)$ and therefore the $i_{r}$ th moment of $Y(d, 0)$ is less than $M_{i_{r}}(X)-\delta$. Let us now consider two sequences of positive numbers $\left\{d_{\nu}\right\}$ and $\left\{\eta_{\nu}\right\}\left(\nu=1, \cdots\right.$, ad inf.) such that $\lim d_{\nu}=\infty$, $\lim \eta_{\nu}=0, \lim d_{\nu}^{t_{\nu}-1} \eta_{\nu}=0$, and $\lim d_{\nu}^{i_{r}} \eta_{\nu}=\infty$. It is obvious that $\lim _{\nu=\infty} M_{i_{n}}\left(d_{\nu}, \eta_{\nu}\right)$ $=M_{i_{n}}^{\prime}$ for $n=1, \cdots, r-1$. Hence for sufficiently large $\nu$ the $(r-1)$-tuple $M_{i_{1}}\left(d_{\nu}, \bar{\eta}_{\nu}\right), \cdots, M_{i_{r-1}}\left(d_{\nu}, \bar{\eta}_{\nu}\right)$ lies in the $\delta$-neighborhood of $M$ for any positive $\bar{\eta}_{\nu} \leqq \eta_{\nu}$. On the other hand the $i_{r}$ th moment of $Y\left(d_{\nu}, \eta_{\nu}\right)$ converges towards infinity. If $\alpha$ denotes an arbitrary number greater than $M_{i_{r}}(X)-\delta$, then for sufficiently large $\nu$ the $i_{r}$ th moment of $Y\left(d_{\nu}, \eta_{\nu}\right)$ will be greater than $\alpha$. Since the $i_{r}$ th moment of $Y\left(d_{\nu}, 0\right)$ is less than $\alpha$, there exists a number $\bar{\eta}_{\nu}<\eta_{\nu}$ such that the $i_{r}$ th moment of $Y\left(d_{\nu}, \bar{\eta}_{v}\right)$ is equal to $\alpha$. This proves the Lemma $B_{k}$.

Now we shall prove $A_{k+1}$ by means of $A_{k}$ and $B_{k}$. Denote by $M_{i_{1}}, \cdots, M_{i_{k+1}}$ the moments of the orders $i_{1}<\cdots<i_{k+1}$ of a certain chance variable $X$. According to $A_{k}$ there exists a chance variable $Y$ which is characteristic relative to $M_{i_{1}}, \cdots, M_{i_{k}}$. If $Y$ is degenerate, then according to Proposition 6, $X$ must be equal to $Y$ and $Y$ is therefore characteristic also relative to $M_{i_{1}}, \cdots, M_{i_{k+1}}$. Hence in this case $A_{k+1}$ is proved. We have to consider only the case that $Y$ is not degenerate. Hence the degree of $Y$ is equal to $(k+1) / 2$. On account of Proposition $8, M_{i_{k+1}}(Y) \leqq M_{i_{k+1}}$. If $M_{i_{k+1}}(Y)=M_{i_{k+1}}$, then $Y$ is characteristic relative to $M_{i_{1}}, \cdots, M_{i_{k+1}}$ and $A_{k+1}$ is proved. We have to deal only with the case that $M_{i_{k+1}}(Y)<M_{i_{k+1}}$. Denote by $d_{0}$ the greatest positive value for which $P\left(Y=d_{0}\right)>0$. Consider the chance variable $Y_{d, \epsilon}$ which is characteristic relative to

$$
M_{i_{1}}(d, \epsilon)=\frac{M_{i_{1}}-d^{i_{1}} \epsilon}{1-\epsilon}, \cdots, M_{i_{k}}(d, \epsilon)=\frac{M_{i_{k}}-d^{i_{k} \cdot \epsilon}}{1-\epsilon},
$$

where $d>d_{0}$. On account of $B_{k}, Y_{d, \epsilon}$ exists for sufficiently small $\epsilon$. According to Proposition 12, $\lim _{\epsilon=0} Y_{d, \epsilon}=Y$. Hence for sufficiently small values of $\epsilon$, 
$Y_{d, \epsilon}$ is not degenerate. From Proposition 12 and $B_{k}$ it follows easily that for any given $d$ the set of values $\epsilon$ for which $Y_{d, \epsilon}$ exists and is not degenerate is an open set. Hence there exists a smallest value $\epsilon(d)$ for which $Y_{d, \epsilon(d)}$ is degenerate or does not exist. First we shall prove

LeMma 1. $P\left(Y_{d, \epsilon}=d\right)=0$ for $d>d_{0}$ and for any $\epsilon$ for which $Y_{d, \epsilon}$ exists.

Let us suppose that there exists a value $d>d_{0}$ and a positive $\epsilon$ such that $Y_{d, \epsilon}$ exists and $P\left(Y_{d, \epsilon}=d\right)>0$. Consider the chance variable $\bar{Y}_{d, \epsilon}$ defined as follows:

$$
\begin{aligned}
& P\left(\bar{Y}_{d, \epsilon}=d\right)=P\left(Y_{d, \epsilon}=d\right) \cdot(1-\epsilon)+\epsilon ; \\
& P\left(\bar{Y}_{d, \epsilon}=x\right)=P\left(Y_{d, \epsilon}=x\right) \cdot(1-\epsilon),
\end{aligned}
$$$$
\text { for } x \neq d \text {. }
$$

It is obvious that $M_{i_{\nu}}\left(\bar{Y}_{d, \epsilon}\right)=M_{i_{\nu}}(\nu=1, \cdots, k)$ and the degree of $\bar{Y}_{d, \epsilon}$ is not greater than the degree of $Y_{d, \epsilon}$. Hence $\bar{Y}_{d, \epsilon}$ is characteristic relative to $M_{i_{1}}, \cdots, M_{i_{k}}$. According to Proposition $7, \bar{Y}_{d, \epsilon}$ must be equal to $Y$, which is not the case, since $P\left(\bar{Y}_{d, \epsilon}=d\right)>0$ and $P(Y=d)=0$. Hence we have a contradiction and the assumption $P\left(Y_{d, \epsilon}=d\right)>0$ is proved to be an absurdity.

We shall now prove the

Lemma 2. If $d>d_{0}$ then for each $\epsilon<\epsilon(d), P\left(Y_{d, \epsilon} \geqq d\right)=0$.

In fact $Y_{d, 0}=Y$ and therefore $P\left(Y_{d, 0} \geqq d\right)=0$. On account of Proposition $12, Y_{d, \epsilon}$ is a continuous function of $\epsilon$ in the half open interval [0, $\left.\epsilon(d)\right)$. Hence if there exists a value $\epsilon^{\prime}<\epsilon(d)$ for which $P\left(Y_{d, \epsilon^{\prime}} \geqq d\right)>0$, then $P\left(Y_{d, \epsilon}=d\right)>0$ must hold for a certain value $\epsilon=\epsilon^{\prime \prime} \leqq \epsilon^{\prime}$, in contradiction to Lemma 1 .

Lemma 3. For $d>d_{0}, Y_{d, \epsilon(d)}$ exists and $P\left(Y_{d, \epsilon(d)} \geqq d\right)=0$.

Denote by $\left\{\epsilon_{n}\right\}$ a sequence of positive numbers for which $\epsilon_{n}<\epsilon(d)$ and $\lim \epsilon_{n}=\epsilon(d)$. Consider the corresponding sequence $\left\{Y_{d, \epsilon_{n}}\right\}$ of chance variables. On account of Proposition 10 there exists a convergent subsequence $\left\{Y_{d, \epsilon_{n}^{\prime}}\right\}$ of the sequence $\left\{Y_{d, \epsilon_{n}}\right\}$. Denote $\lim _{n=\infty} Y_{d, \epsilon_{n}^{\prime}}$ by $Y_{d}$. Since according to Lemma $1, P\left(Y_{d, \epsilon} \geqq d\right)=0,\left\{M_{r}\left(Y_{d, \epsilon_{n}^{\prime}}\right)\right\}$ is bounded for any integer $r$. Hence we have on account of Proposition 9

$$
\lim _{n=\infty} M_{r}\left(Y_{d, \epsilon_{n}^{\prime}}\right)=M_{r}\left(Y_{d}\right)
$$

for any integer $r$. Then from

$$
M_{i_{\nu}}=\frac{M_{i_{\nu}}-d^{i_{\nu} \cdot \epsilon_{n}}}{1-\epsilon_{n}}, \quad \quad \nu=1, \cdots, k ; n=1, \cdots, \text { ad inf., }
$$

it follows that

$$
M_{i_{\nu}}\left(Y_{d}\right)=\lim \frac{M_{i_{\nu}}-d^{i_{\nu}} \cdot \epsilon_{n}^{\prime}}{1-\epsilon_{n}^{\prime}}=\frac{M_{i_{\nu}}-d^{i_{\boldsymbol{v}}}(d)}{1-\epsilon(d)}, \quad \nu=1, \cdots, k .
$$


Since $Y_{d}$ is characteristic relative to the above moments, $Y_{d, \epsilon(d)}$ exists and is equal to $Y_{d}$. From $P\left(Y_{d, \epsilon_{n}} \geqq d\right)=0$ and $\lim Y_{d, \epsilon_{n}^{\prime}}=Y_{d, \epsilon(d)}$ it follows that $P\left(Y_{d, \epsilon(d)}>d\right)=0$. Since on account of Lemma 1, $P\left(Y_{d, \epsilon(d)}=d\right)=0$, we have $P\left(Y_{d, \epsilon(d)} \geqq d\right)=0$.

Now we are able to prove

LEMMa 4. Besides $\epsilon(d)$ no other value $\epsilon^{\prime}$ can be given for which $Y_{d, \epsilon^{\prime}}$ exists and is degenerate provided $d>d_{0}$.

Let us suppose that there exists a positive $\epsilon^{\prime} \neq \epsilon(d)$ for which $Y_{d, \epsilon^{\prime}}$ exists and is degenerate. Consider the chance variables $\bar{Y}_{d, \epsilon^{\prime}}$ and $\bar{Y}_{d, \epsilon(d)}$ defined as follows:

$P\left(\bar{Y}_{d, \epsilon}=d\right)=\epsilon ; P\left(\bar{Y}_{d, \epsilon}=x\right)=P\left(Y_{d, \epsilon}=x\right) \cdot(1-\epsilon)$, for $x \neq d ; \epsilon=\epsilon^{\prime}, \epsilon(d)$.

Since $Y_{d, \epsilon^{\prime}}$ and $Y_{d, \epsilon(d)}$ are degenerate, their degrees are less than or equal to $(k+1) / 2-1 / 2=k / 2$. The degree of $\bar{Y}_{d, \epsilon^{\prime}}$ and that of $\bar{Y}_{d, \epsilon(d)}$ are obviously not greater than $k / 2+1$. Hence on account of Proposition 4,

$$
D(x)=P\left(\bar{Y}_{d, \epsilon^{\prime}}<x\right)-P\left(\bar{Y}_{d, \epsilon(d)}<x\right)
$$

has at most $2(k / 2+1)-3=k-1$ changes in sign. Since $M_{i_{\nu}}\left(\bar{Y}_{d, \epsilon^{\prime}}\right)$ $=M_{i_{\nu}}\left(\bar{Y}_{d, \epsilon(d)}\right)=M_{i_{\nu}}(\nu=1, \cdots, k), D(x)$ must be identically equal to zero on account of Proposition 5. But $D(x)$ cannot be identically equal to zero since $P\left(\bar{Y}_{d, \epsilon^{\prime}}=d\right)=\epsilon^{\prime}, P\left(\bar{Y}_{d, \epsilon(d)}=d\right)=\epsilon(d)$ and $\epsilon^{\prime} \neq \epsilon(d)$. Hence the assumption that there exists an $\epsilon^{\prime} \neq \boldsymbol{\epsilon}(d)$ for which $Y_{d, \epsilon^{\prime}}$ exists and is degenerate is proved to be an absurdity.

Let us consider a sequence of numbers $\left\{d_{n}\right\}(n=1, \cdots$, ad inf.) for which $d_{n}>d_{0}$ and $\lim d_{n}=d>d_{0}$. We shall show that $\lim \epsilon\left(d_{n}\right)=\epsilon(d)$ and $\lim Y_{d_{n}, \epsilon\left(d_{n}\right)}$ $=Y_{d, \epsilon(d)}$. In order to prove $\lim Y_{d_{n}, \epsilon\left(d_{n}\right)}=Y_{d, \epsilon(d)}$, we have only to show on account of Proposition 11 that for each convergent subsequence $\left\{Y_{d_{n^{\prime}}, \epsilon\left(d_{n^{\prime}}\right)}\right\}$ of the sequence $\left\{Y_{d_{n}, \epsilon\left(d_{n}\right)}\right\}$

$$
\lim Y_{d_{n^{\prime}, \in\left(d_{n^{\prime}}\right)}}=Y_{d, \epsilon(d)} .
$$

Denote $\lim Y_{d_{n^{\prime}, \epsilon\left(d_{n^{\prime}}\right)}}$ by $Y^{*}$. Since $P\left[Y_{{d_{n^{\prime}}, \epsilon\left(d_{n^{\prime}}\right)}}>d_{n}{ }^{\prime}\right]=0,\left\{M_{r}\left(Y_{d_{n^{\prime}}, \epsilon\left(d_{n^{\prime}}\right)}\right)\right\}$ is bounded for any $r$. Hence we have on account of Proposition 9

$$
\lim _{n=\infty} M_{r}\left(Y_{\left.d_{n^{\prime}, e\left(d_{n^{\prime}}\right)}\right)}\right)=M_{r}\left(Y^{*}\right)
$$

for any positive integer $r$. Since

$$
M_{i_{\nu}}\left(Y_{\left.d_{n^{\prime}, \epsilon\left(d_{n}\right)}\right)}\right)=\frac{M_{i_{\nu}}-\left(d_{n}^{\prime}\right)^{i \boldsymbol{i} \cdot \epsilon}\left(d_{n}^{\prime}\right)}{1-\epsilon\left(d_{n}^{\prime}\right)}
$$

converges with increasing $n$ and since $\lim \left(d_{n}{ }^{\prime}\right)^{i_{\nu}}=d^{i_{\nu}}>d_{0}{ }^{i_{\nu}}>M_{i_{\nu}}$, the sequence 
$\left\{\epsilon\left(d_{n}{ }^{\prime}\right)\right\}$ must also converge. Denote $\lim \epsilon\left(d_{n}{ }^{\prime}\right)$ by $\epsilon^{*}$. Then $Y^{*}$ is characteristic relative to

$$
M_{i_{1}}\left(d, \epsilon^{*}\right), \cdots, M_{i_{k}}\left(d, \epsilon^{*}\right) ;
$$

that is to say, $Y^{*}$ is equal to $Y_{d, \epsilon^{*} \text {. Since }} Y_{d, \epsilon^{*}}=\lim Y_{d_{n^{\prime}}, \epsilon\left(d_{n^{\prime}}\right)}$ and $Y_{d_{n^{\prime}, \epsilon\left(d_{n^{\prime}}\right)}}$ is degenerate, $Y_{d, e^{*}}$ must also be degenerate. Then according to Lemma 4, $\epsilon^{*}=\epsilon(d)$ and therefore $Y_{d, \epsilon^{*}}$ is equal to $Y_{d, \epsilon(d)}$. Hence our statement that $\lim Y_{d_{n}, \epsilon\left(d_{n}\right)}=Y_{d, \epsilon(d)}$ is proved. Since according to Lemma 3, $P\left(Y_{d_{n}, \epsilon\left(d_{n}\right)}\right.$ $\left.\geqq d_{n}\right)=0$ and therefore $M_{r}\left(Y_{d_{n}, \epsilon\left(d_{n}\right)}\right)$ is bounded for any integer $r$, we have on account of Proposition 9

$$
\lim _{n=\infty} M_{r}\left(Y_{d_{n}, \epsilon\left(d_{n}\right)}\right)=M_{r}\left(Y_{d, \epsilon(d)}\right) .
$$

From this it follows that $\lim \epsilon\left(d_{n}\right)=\epsilon(d)$ and that the moments of $Y_{d, \epsilon(d)}$ are continuous functions of $d$ for $d>d_{0}$.

Denote by $\bar{Y}_{d, \epsilon}$ the chance variable defined as follows:

$$
P\left(\bar{Y}_{d, \epsilon}=d\right)=\epsilon ; \quad P\left(\bar{Y}_{d, \epsilon}=x\right)=P\left(Y_{d, \epsilon}=x\right) \cdot(1-\epsilon), \quad \text { for } x \neq d .
$$

It is obvious that

$$
M_{i_{\nu}}\left(\bar{Y}_{d, \epsilon}\right)=M_{i_{\nu}}, \quad \quad \nu=1, \cdots, k .
$$

In order to show that $\lim _{d=\infty} M_{i_{k_{1}}}\left(\bar{Y}_{d, \epsilon(d)}\right)=\infty$ we have only to show that for any sequence $\left\{d_{n}\right\}$ for which $\lim d_{n}=\infty, \dot{d}_{n} i_{k} \cdot \epsilon\left(d_{n}\right)$ does not converge towards zero. In order to prove the latter statement, let us assume that $\lim d_{n}{ }^{i_{k}} \epsilon\left(d_{n}\right)=0$ and $\lim d_{n}=\infty$. It is obvious that $\lim d_{n}{ }^{i_{\nu} \epsilon}\left(d_{n}\right)=0$ for $\nu=1,2, \cdots, k$. Hence

$$
\lim _{d=\infty} M_{i_{\nu}}\left(Y_{d, \epsilon(d)}\right)=M_{i_{\nu}}, \quad \quad \nu=1, \cdots, k .
$$

Since $Y$ is characteristic relative to $M_{i_{1}}, \cdots, M_{i_{k}}$, we have, on account of Proposition 12, $\lim Y_{d_{n}, \epsilon\left(d_{n}\right)}=Y$. But this is not possible since $Y_{d_{n}, \epsilon\left(d_{n}\right)}$ is degenerate and therefore $\lim Y_{d_{n}, \epsilon\left(d_{n}\right)}$ must also be degenerate and consequently cannot be equal to $Y$ which is not degenerate. Hence we have $\lim M_{i_{k+1}}\left(\bar{Y}_{d_{n}, \epsilon\left(d_{n}\right)}\right)=\infty$.

On account of Proposition 10 there exists a sequence $\left\{d_{n}\right\}$ such that $d_{n}>d_{0}$, lim $d_{n}=d_{0}$, and the sequence $\left\{\bar{Y}_{d_{n}, \epsilon\left(d_{n}\right)}\right\}$ is convergent. Denote $\lim \bar{Y}_{d_{n}, \epsilon\left(d_{n}\right)}$ by $Y^{*}$. Since $M_{i_{\nu}}\left(\bar{Y}_{d_{n}, \epsilon\left(d_{n}\right)}\right)=M_{i_{\nu}}(\nu=1, \cdots, k)$ and $P\left(\bar{Y}_{d_{n}, \epsilon\left(d_{n}\right)}\right.$ $\left.>d_{n}\right)=0$, we have, on account of Proposition $9, M_{i_{\nu}}\left(Y^{*}\right)=M_{i_{\nu}}(\nu=1, \cdots, k)$. The degree of $Y_{d, \epsilon(d)}$ is less than or equal to $k / 2$ and therefore the degree of $\bar{Y}_{d, \epsilon(d)}$ is less than or equal to $k / 2+1$. Hence also the degree of $Y^{*}$ is less than or equal to $k / 2+1$. Now we shall show that $P\left(Y^{*}=d_{0}\right)>0$. Let us assume that $P\left(Y^{*}=d_{0}\right)=0$. Then $\lim \epsilon\left(d_{n}\right)$ must be equal to zero. Hence $\lim M_{i_{\nu}}\left(Y_{d_{n}, \epsilon\left(d_{n}\right)}\right)$ 
$=M_{i_{\nu}}(\nu=1, \cdots, k)$, and then on account of Proposition 12, $Y_{d_{n}, \epsilon\left(d_{n}\right)}$ must converge towards $Y$ which cannot be the case since $Y_{d_{n}, \epsilon\left(d_{n}\right)}$ is degenerate and $Y$ is not degenerate. Hence $P\left(Y^{*}=d_{0}\right)>0$ is proved. Since $P\left(Y=d_{0}\right)>0, P\left(Y^{*}=d_{0}\right)>0$, we get from Proposition 4 that the number of changes in sign of $D(x)=P\left(Y^{*}<x\right)-P(Y<x)$ is less than or equal to $2(k / 2+1)-3=k-1$. Since $M_{i_{\nu}}\left(Y^{*}\right)=M_{i_{\nu}}(Y)(\nu=1, \cdots, k)$, on account of Proposition 5, $D(x)$ must be identically equal to zero, that is to say, $Y^{*}=Y$. Hence

$$
\lim M_{i_{k+1}}\left(\bar{Y}_{d_{n}, \epsilon\left(d_{n}\right)}\right)=M_{i_{k+1}}\left(Y^{*}\right)=M_{i k_{+1}}(Y)<M_{i_{k+1}} .
$$

Since $\lim _{d=\infty} M_{i_{k+1}}\left(\bar{Y}_{d, \epsilon(d)}\right)=\infty$ and $M_{i_{k+1}}\left(\bar{Y}_{d, \epsilon(d)}\right)$ is a continuous function of $d$, there exists a value $d^{\prime}$ such that $M_{i_{k+1}}\left(\bar{Y}_{d^{\prime}, \epsilon\left(d^{\prime}\right)}\right)=M_{i_{k+1}}$. The degree of $\bar{Y}_{d^{\prime}, \epsilon\left(d^{\prime}\right)}$ is less than or equal to $k / 2+1$, and therefore $\bar{Y}_{d^{\prime}, \epsilon\left(d^{\prime}\right)}$ is characteristic relative to $M_{i_{1}}, \cdots, M_{i_{k+1}}$. This proves $A_{k+1}$, and therefore Proposition 13 is also proved.

Since Proposition 13 is proved, $B_{k}$ is also proved for any positive integer $k$. Hence we can formulate

PROPOSITION 14. If the chance variable which is characteristic relative to the moments $M_{i_{1}}, \cdots, M_{i_{k}}$ is not degenerate, then there exists a positive $\delta$ such that any $k$-tuple $M_{i_{1}}^{\prime}, \cdots, M_{i_{k}}^{\prime}$ in the $\delta$-neighborhood of the k-tuple $M_{i_{1}}, \cdots, M_{i_{k}}$ can be realized as moments of the orders $i_{1}, \cdots, i_{k}$.

4. Solution of Problem 1. Denote by $M_{i_{1}}, \cdots, M_{i_{k}}$ the moments of the order $i_{1}<i_{2}<\cdots<i_{k}$ of a certain chance variable $X$. Denote by $X^{\prime}$ the characteristic chance variable relative to $M_{i_{1}}, \cdots, M_{i_{k}}$. If $X^{\prime}$ is degenerate, then according to Proposition 6 no chance variable $Y \neq X^{\prime}$ exists for which $M_{i_{\nu}}(Y)=M_{i_{\nu}}\left(X^{\prime}\right)(\nu=1, \cdots, k)$. Hence the sharp lower and the sharp upper limits of $P(X<d)$ are equal to $P\left(X^{\prime}<d\right)$ and our problem is solved. Throughout the following development we shall suppose that $X^{\prime}$ is not degenerate.

Consider the $k$-tuple of values

$$
M_{i_{v}}(d, \lambda)=\frac{M_{i_{\nu}}-d^{i \nu} \cdot \lambda}{1-\lambda}, \quad \quad \nu=1, \cdots, k,
$$

where $d>0,0 \leqq \lambda<1$. According to Proposition 14 the $k$-tuple $M_{i_{1}}(d, \lambda), \cdots$, $M_{i_{k}}(d, \lambda)$ can be realized as moments for sufficiently small values of $\lambda$. Denote by $Y(d, \lambda)$ the characteristic chance variable relative to the moments $M_{i_{1}}(d, \lambda), \cdots, M_{i_{k}}(d, \lambda)$. Denote further by $\bar{Y}(d, \lambda)$ the arithmetic chance variable defined as follows:

$$
\begin{aligned}
& P[\bar{Y}(d, \lambda)=d]=P[Y(d, \lambda)=d](1-\lambda)+\lambda, \\
& P[\bar{Y}(d, \lambda)=x]=P[Y(d, \lambda)=x](1-\lambda),
\end{aligned}
$$

for $x \neq d$. 
It is obvious that

$$
M_{i_{\nu}}[\bar{Y}(d, \lambda)]=M_{i_{\nu}}, \quad \quad \nu=1, \cdots, k .
$$

From Proposition 14 it follows that for any given $d>0$ the set $\Omega$ of values of $\lambda$ for which the characteristic chance variable relative to the moments $M_{i_{1}}(d, \lambda), \cdots, M_{i_{k}}(d, \lambda)$ exists and is not degenerate is an open set. Denote by $\lambda_{d}$ the smallest positive value not belonging to $\Omega$.

As is well known, $M_{r}{ }^{s / r} \leqq M_{s}$ for any integer $r<s$, and the equality sign holds only if the chance variable is of the degree less than or equal to 1 . Since for $\lambda<\lambda_{d}$ the characteristic chance variable $Y(d, \lambda)$ is not degenerate, we have

$$
\left[M_{i_{\mu}}(d, \lambda)\right]^{i_{\nu} / i_{\mu}}<M_{i_{\nu}}(d, \lambda), \quad \text { for } \mu<\nu, \mu<k, \nu \leqq k .
$$

From these inequalities and from the fact that negative moments are not possible, it follows easily that if $k \geqq 3, \lambda_{d}<1$ for any positive value $d$. If $k=2$, $\lambda_{d}$ can be equal to 1 only if $d=M_{i_{1}}{ }^{1 / i_{1}}$.

Now we shall prove

Proposition 15. Denote by $\left\{\lambda_{n}\right\}(n=1,2, \cdots$, ad inf.) a sequence of positive values such that $\lambda_{n}<\lambda_{d}$ and $\lim \lambda_{n}=\lambda_{d}$. Then $\lim Y\left(d, \lambda_{n}\right)$ exists and is equal to the chance variable $Y_{d}$ which is characteristic relative to the $k-1$ moments $M_{i_{1}}\left(d, \lambda_{d}\right), \cdots, M_{i_{k-1}}\left(d, \lambda_{d}\right)$. If $Y_{d}$ is not degenerate, then $Y_{d}$ is characteristic also relative to the $k$ moments $M_{i_{1}}\left(d, \lambda_{d}\right), \cdots, M_{i_{k}}\left(d, \lambda_{d}\right)$.

According to Proposition 10 there exists a convergent subsequence of the sequence $\left\{Y\left(d, \lambda_{n}\right)\right\}\left(n=1, \cdots\right.$, ad inf.). Denote by $\left\{Y\left(d, \lambda_{n}^{\prime}\right)\right\}$ a convergent subsequence of $\left\{Y\left(d, \lambda_{n}\right)\right\}$ and denote $\lim Y\left(d, \lambda_{n}^{\prime}\right)$ by $Y^{*}$. If $Y\left(d, \lambda_{d}\right)$ exists, then according to Proposition $12, Y^{*}$ must be equal to $Y\left(d, \lambda_{d}\right)$. Since $Y\left(d, \lambda_{d}\right)$ is degenerate, $Y\left(d, \lambda_{d}\right)$ is characteristic also relative to the $k-1 \mathrm{mo-}$ ments $M_{i_{1}}\left(d, \lambda_{d}\right), \cdots, M_{i_{k-1}}\left(d, \lambda_{d}\right)$. Hence $Y^{*}=Y\left(d, \lambda_{d}\right)=Y_{d}$. We have now to consider the case that $Y\left(d, \lambda_{d}\right)$ does not exist, that is to say, the $k$-tuple $M_{i_{1}}\left(d, \lambda_{d}\right), \cdots, M_{i_{k}}\left(d, \lambda_{d}\right)$ cannot be realized as moments. On account of Proposition 9,

$$
M_{i_{\nu}}\left(Y^{*}\right)=M_{i_{\nu}}\left(d, \lambda_{d}\right), \quad \nu=1, \cdots, k-1 .
$$

Since the $k$-tuple $M_{i_{1}}\left(d, \lambda_{d}\right), \cdots, M_{i_{k}}\left(d, \lambda_{d}\right)$ cannot be realized,

$$
M_{i_{k}}\left(Y^{*}\right) \neq M_{i_{k}}\left(d, \lambda_{d}\right) \text {. }
$$

From this it follows on account of Proposition 9 that $\left\{M_{r}\left[Y\left(d, \lambda_{n}^{\prime}\right)\right]\right\}$ $\left(n=1,2, \cdots\right.$, ad inf.) is not bounded for any integer $r>i_{k}$. Hence there exists a subsequence $\left\{Y\left(d, \lambda_{n}^{\prime \prime}\right)\right\}$ such that $\lim _{n=\infty} M_{r}\left[Y\left(d, \lambda_{n}^{\prime \prime}\right)\right]=\infty$. Denote by $\alpha_{n}$ the greatest positive value for which $P\left[Y\left(d, \lambda_{n}^{\prime \prime}\right)=\alpha_{n}\right]>0$. It is obvious that $\lim \alpha_{n}=\infty$ and 


$$
\lim P\left[Y\left(d, \lambda_{n}^{\prime \prime}\right)=\alpha_{n}\right]=0 .
$$

Hence the degree of $Y^{*}$ must be less than or equal to $(k+1) / 2-1=(k-1) / 2$. Since $M_{i_{\nu}}\left(Y^{*}\right)=M_{i_{\nu}}\left(d, \lambda_{d}\right) \quad(\nu=1, \cdots, k-1), Y^{*}$ is characteristic and degenerate relative to $M_{i_{1}}\left(d, \lambda_{d}\right), \cdots, M_{i_{k-1}}\left(d, \lambda_{d}\right)$. That is to say, $Y^{*}=Y_{d}$ and $Y_{d}$ is degenerate.

Hence we have proved that in any case the limit of a convergent subsequence of $\left\{Y\left(d, \lambda_{n}\right)\right\}$ is equal to $Y_{d}$. From this fact it follows on account of Proposition 11 that $\lim Y\left(d, \lambda_{n}\right)=Y_{d}$. As we have shown, $Y_{d}=Y\left(d, \lambda_{d}\right)$ if $Y\left(d, \lambda_{d}\right)$ exists, and $Y_{d}$ is degenerate if $Y\left(d, \lambda_{d}\right)$ does not exist. Hence Proposition 15 is proved.

Proposition 16. $P\left(Y_{d}=d\right)=0$, where $Y_{d}$ denotes the characteristic chance variable relative to the $k-1$ moments $M_{i_{1}}\left(d, \lambda_{d}\right), \cdots, M_{i_{k-1}}\left(d, \lambda_{d}\right)$.

Let us suppose $P\left(Y_{d}=d\right)>0$. Denote by $\bar{Y}_{d}$ the chance variable defined as follows:

$$
\begin{aligned}
& P\left(\bar{Y}_{d}=d\right)=P\left(Y_{d}=d\right) \cdot\left(1-\lambda_{d}\right)+\lambda_{d} ; \\
& P\left(\bar{Y}_{d}=x\right)=P\left(Y_{d}=x\right) \cdot\left(1-\lambda_{d}\right),
\end{aligned}
$$

for $x \neq d$.

If $Y\left(d, \lambda_{d}\right)$ exists, then $M_{i_{\nu}}\left(Y_{d}\right)=M_{i_{\nu}}\left(d, \lambda_{d}\right)(\nu=1, \cdots, k)$ and therefore $M_{i_{\nu}}\left(\bar{Y}_{d}\right)=M_{i_{\nu}}(\nu=1, \cdots, k)$. The degree of $\bar{Y}_{d}$ is equal to the degree of $Y_{d}$. Hence $\bar{Y}_{d}$ is characteristic and degenerate relative to $M_{i_{1}}, \cdots, M_{i_{k}}$, in contradiction to our assumption that the characteristic chance variable relative to $M_{i_{1}}, \cdots, M_{i_{k}}$ is not degenerate. If $Y\left(d, \lambda_{d}\right)$ does not exist, then $Y_{d}$ is degenerate. That is to say, the degree of $Y_{d}$ is less than or equal to $(k-1) / 2$. Since $M_{i_{\nu}}\left(\bar{Y}_{d}\right)=M_{i_{\nu}}(\nu=1, \cdots, k-1)$ and the degree of $\bar{Y}_{d}$ is equal to the degree of $Y_{d}, \bar{Y}_{d}$ is characteristic and degenerate relative to the $k-1$ moments $M_{i_{1}}, \cdots, M_{i_{k-1}}$. But on account of our assumption that the characteristic chance variable relative to $M_{i_{1}}, \cdots, M_{i_{k}}$ is not degenerate, from Proposition 6 it follows that the characteristic chance variable relative to $M_{i_{1}}, \cdots, M_{i_{k-1}}$ also cannot be degenerate. Hence we have a contradiction and the assumption that $P\left(Y_{d}=d\right)>0$ is proved to be an absurdity.

Proposition 17. Denote by $\left\{d_{n}\right\}$ and $\left\{\lambda_{n}\right\}(n=1,2, \cdots$, ad inf.) two sequences of positive values such that $\lim d_{n}=d>0, \lim \lambda_{n}=\lambda<\lambda_{d}$. Then $\lim Y\left(d_{n}, \lambda_{n}\right)=Y(d, \lambda)$.

On account of Proposition 14, $Y\left(d_{n}, \lambda_{n}\right)$ exists for almost every $n$. Since $\lim M_{i_{\nu}}\left[Y\left(d_{n}, \lambda_{n}\right)\right]=M_{i_{\nu}}[Y(d, \lambda)]$, we have on account of Proposition 12 that $\lim Y\left(d_{n}, \lambda_{n}\right)=Y(d, \lambda)$.

Proposition 18. The sharp lower limit $a_{d}$ of $P(X<d)$ is equal to $P\left(\bar{Y}_{d}<d\right)$, and the sharp upper limit $b_{d}$ of $P(X<d)$ is equal to $P\left(\bar{Y}_{d} \leqq d\right)$ where $\bar{Y}_{d}$ denotes 
the arithmetic chance variable defined as follows:

$$
P\left(\bar{Y}_{d}=d\right)=\lambda_{d} ; \quad P\left(\bar{Y}_{d}=x\right)=P\left(Y_{d}=x\right) \cdot\left(1-\lambda_{d}\right), \quad \text { for } x \neq d .
$$

We shall consider two cases.

(1) $Y_{d}$ is not degenerate. Hence the degree of $Y_{d}$ is equal to $k / 2$. According to Proposition 15, $Y_{d}$ is characteristic also relative to $M_{i_{1}}\left(d, \lambda_{d}\right), \cdots, M_{i_{k}}\left(d, \lambda_{d}\right)$. Hence

$$
M_{i_{\nu}}\left(\bar{Y}_{d}\right)=M_{i_{\nu}}, \quad \nu=1, \cdots, k .
$$

Since, according to Proposition 16, $P\left(Y_{d}=d\right)=0$, the degree of $\bar{Y}_{d}$ is obviously equal to $k / 2+1$. Let us suppose that there exists a chance variable $X$ such that $M_{i_{\nu}}(X)=M_{i_{\nu}}(\nu=1, \cdots, k)$ and $P(X<d)<P\left(\bar{Y}_{d}<d\right)$. Denote by $\alpha$ the greatest number less than $d$ for which $P\left(\bar{Y}_{d}=\alpha\right)>0$. It is obvious that $D(x)=P(X<x)-P\left(\bar{Y}_{d}<d\right)$ has no change in sign in the interior of the interval $[\alpha, d]$. If $D(x)$ is identically zero in the interior of $[\alpha, d]$, then $D(x)$ has no change in sign at $\alpha$. If $D(x)$ is not identically zero in the interior of $[\alpha, d]$ and if $P(X \leqq d) \leqq P\left(\bar{Y}_{d} \leqq d\right)$, then $D(x)$ has no change in sign at $d$. Finally if $P(X \leqq d)>P\left(\bar{Y}_{d} \leqq d\right)$ and if $\beta$ denotes the smallest value greater than $d$ for which $P\left(\bar{Y}_{d}=\beta\right)>0$, then $D(x)$ has no change in sign in the interior of the interval $[d, \beta]$. From this fact it follows easily that the number of changes in sign of $D(x)$ cannot exceed $2(k / 2+1)-3=k-1$. Since $M_{i_{\nu}}(X)$ $=M_{i_{\nu}}(\bar{Y})(\nu=1, \cdots, k)$, this is in contradiction to Proposition 5 . Hence the assumption $P(X<d)<P\left(\bar{Y}_{d}<d\right)$ is proved to be an absurdity. Now let us assume that there exists a chance variable $X$ such that $M_{i_{\nu}}(X)=M_{i_{\nu}}$ $(\nu=1, \cdots, k)$ and $P(X<d)>P\left(\bar{Y}_{d} \leqq d\right)$. Denote by $\beta$ the smallest number greater than $d$ for which $P\left(\bar{Y}_{d}=\beta\right)>0$. It is obvious that $D(x)=P(X<x)$ $-P\left(\bar{Y}_{d}<x\right)$ has no change in sign at the point $d$ and also no change in sign in the interior of the interval $[d, \beta]$. Hence the number of changes in sign of $D(x)$ cannot exceed $2(k / 2+1)-3=k-1$. But this is in contradiction to Proposition 5, and the assumption $P(X<d)>P\left(\bar{Y}_{d} \leqq d\right)$ therefore is proved to be an absurdity.

We now have to show that the limits $P\left(\bar{Y}_{d}<d\right)$ and $P\left(\bar{Y}_{d} \leqq d\right)$ are sharp. Since $M_{i_{\nu}}\left(\bar{Y}_{d}\right)=M_{i_{\nu}}(\nu=1, \cdots, k)$, the lower limit $P\left(\bar{Y}_{d}<d\right)$ is evidently sharp. Denote by $\left\{d_{n}\right\}(n=1,2, \cdots$, ad inf.) a sequence of positive numbers for which $d_{n}<d$ and $\lim d_{n}=d$. Denote by $\lambda$ some value less than $\lambda_{d}$. It is obvious that $Y\left(d_{n}, \lambda\right)$ exists for almost every $n$ and that on account of Proposition 12, $\lim _{n=\infty} Y\left(d_{n}, \lambda\right)=Y(d, \lambda)$. Since $P\left(Y_{d}=d\right)=0$ the function $P\left(Y_{d}<x\right)$ is constant in the neighborhood of $x=d$. Then from $\lim _{\lambda=\lambda_{d}} Y(d, \lambda)$ $=Y_{d}$ it follows that there exists a positive $\eta$ such that

$$
\lim _{\lambda=\lambda_{d}} P[Y(d, \lambda)<d-\eta]=P\left(Y_{d}<d\right) .
$$


Hence to an arbitrarily small positive $\epsilon$ a value $\lambda_{\epsilon}<\lambda_{d}$ can be given such that

$$
P[Y(d, \lambda)<d-\eta]>P\left(Y_{d}<d\right)-\epsilon
$$

for any $\lambda$ greater than $\lambda_{\epsilon}$ and smaller than $\lambda_{d}$. Since $\lim _{n=\infty} Y\left(d_{n}, \lambda\right)=Y(d, \lambda)$,

$$
P\left[Y\left(d_{n}, \lambda\right)<d\right]>P\left(Y_{d}<d\right)-2 \epsilon
$$

for almost every $n$. On account of $d_{n}<d$,

$$
P\left[\bar{Y}\left(d_{n}, \lambda\right)<d\right]=(1-\lambda) P\left[Y\left(d_{n}, \lambda\right)<d\right]+\lambda,
$$

and on account of $P\left(Y_{d}=d\right)=0$,

$$
P\left(\bar{Y}_{d} \leqq d\right)=\left(1-\lambda_{d}\right) P\left(Y_{d}<d\right)+\lambda_{d} .
$$

From (a), (b), and (c), it follows that if we choose $\lambda$ sufficiently near to $\lambda_{d}$, we have

$$
P\left[\bar{Y}\left(d_{n}, \lambda\right)<d\right]>P\left(\bar{Y}_{d} \leqq d\right)-3 \epsilon .
$$

Since $M_{i_{\nu}}\left[\bar{Y}\left(d_{n}, \lambda\right)\right]=M_{i_{\nu}}(\nu=1, \cdots, k)$ and since $\epsilon$ can be chosen arbitrarily small, the upper limit $P\left(\bar{Y}_{d} \leqq d\right)$ is proved to be sharp. Hence Proposition 18 is proved if $Y_{d}$ is not degenerate.

(2) $Y_{d}$ is degenerate. Denote the degree of $Y_{d}$ by $k^{\prime} / 2$ where $k^{\prime}$ denotes a positive integer. It is obvious that $k^{\prime} \leqq k-1$. Since the characteristic chance variable relative to $M_{i_{1}}, \cdots, M_{i_{k}}$ is not degenerate, from Proposition 6 it follows that also the characteristic chance variable relative to $M_{i_{1}}, \cdots, M_{i_{k^{\prime}}}$ is not degenerate. Considering only the moments of the orders $i_{1}, \cdots, i_{k^{\prime}}$ we have case (1) since $Y_{d}$ is obviously characteristic and degenerate relative to the moments $M_{1}\left(d, \lambda_{d}\right), \cdots, M_{i_{k^{\prime}}}\left(d, \lambda_{d}\right)$. Hence $P\left(\bar{Y}_{d}<d\right)$ is the greatest lower and $P\left(\bar{Y}_{d} \leqq d\right)$ is the least upper bound of $P(Z<d)$ where $P(Z<d)$ is formed for all chance variables $Z$ for which $M_{i_{\nu}}(Z)=M_{i_{\nu}}\left(\nu=1, \cdots, k^{\prime}\right)$.

In order to show that the lower limit $P\left(\bar{Y}_{d}<d\right)$ is sharp consider the sequence $\left\{Y\left(d, \lambda_{n}\right)\right\}$ of chance variables where $\lambda_{n}<\lambda_{d}$ and $\lim \lambda_{n}=\lambda_{d}$. Since $\lim Y\left(d, \lambda_{n}\right)=Y_{d}$ and $P\left(Y_{d}=d\right)=0$, we have

$$
\lim _{n=\infty} P\left[Y\left(d, \lambda_{n}\right)<d\right]=P\left(Y_{d}<d\right) .
$$

On account of the fact that

$$
P\left[\bar{Y}\left(d, \lambda_{n}\right)<d\right]=\left(1-\lambda_{n}\right) P\left[Y\left(d, \lambda_{n}\right)<d\right],
$$

and that

$$
P\left(\bar{Y}_{d}<d\right)=P\left(Y_{d}<d\right) \cdot\left(1-\lambda_{d}\right),
$$

we have 


$$
\lim _{n=\infty} P\left[\bar{Y}\left(d, \lambda_{n}\right)<d\right]=P\left(\bar{Y}_{d}<d\right) .
$$

Since $M_{i_{\nu}}\left[\bar{Y}\left(d, \lambda_{n}\right)\right]=M_{i_{\nu}}(\nu=1, \cdots, k)$ the lower limit $P\left(\bar{Y}_{d}<d\right)$ is proved to be sharp. The proof of the fact that also the upper limit $P\left(\bar{Y}_{d} \leqq d\right)$ is sharp is quite analogous to that given in case (1). Hence Proposition 18 is proved.

We can summarize our results in the following

Theorem 1. The moments $M_{i_{1}}, \cdots, M_{i_{j}}$ of the orders $i_{1}, \cdots, i_{j}$ of a certain chance variable $X$ are given. If the chance variable $X^{\prime}$ which is characteristic relative to $M_{i_{1}}, \cdots, M_{i_{j}}$ is degenerate, then the sharp lower limit $a_{d}$ and the sharp upper limit $b_{d}$ are equal to $P\left(X^{\prime}<d\right)$. If $X^{\prime}$ is not degenerate, we have to consider the chance variable $Y_{d}$ which is characteristic relative to $M_{i_{1}}\left(d, \lambda_{d}\right), \cdots$, $M_{i_{j-1}}\left(d, \lambda_{d}\right)$ where

$$
M_{i_{\nu}}(d, \lambda)=\frac{M_{i_{\nu}}-d^{i \nu} \lambda}{1-\lambda}, \quad \quad \nu=1, \cdots, j,
$$

and $\lambda_{d}$ denotes the smallest value $\lambda$ for which $M_{i_{1}}(d, \lambda), \cdots, M_{i_{j}}(d, \lambda)$ cannot be realized as moments, or the characteristic chance variable relative to them is degenerate. The sharp lower limit $a_{d}$ is equal to $P\left(\bar{Y}_{d}<d\right)$ and the sharp upper limit $b_{d}$ is equal to $P\left(\bar{Y}_{d} \leqq d\right)$, where $\bar{Y}_{d}$ denotes the arithmetic chance variable defined as follows:

$$
\begin{aligned}
& P\left(\bar{Y}_{d}=d\right)=P\left(Y_{d}=d\right) \cdot\left(1-\lambda_{d}\right)+\lambda_{d}, \\
& P\left(\bar{Y}_{d}=x\right)=P\left(Y_{d}=x\right) \cdot\left(1-\lambda_{d}\right) \text { for } \quad x \neq d .
\end{aligned}
$$

5. Solution of Problem 2. Denote by $M_{i_{1}}, \cdots, M_{i_{k}}$ the moments of the orders $i_{1}<i_{2}<\cdots<i_{k}$ of a certain chance variable $X$. Consider an integer $i_{k+1}>i_{k}$ and a number $M_{i_{k+1}}$. First we shall deal with the question: what conditions must be satisfied by $M_{i_{k+1}}$ in order that $M_{i_{1}}, \cdots, M_{i_{k+1}}$ can be realized as moments of the orders $i_{1}, \cdots, i_{k+1}$.

If the chance variable $Y$ which is characteristic relative to $M_{i_{1}}, \cdots, M_{i_{k}}$ is degenerate, then on account of Proposition 6 no chance variable $Z \neq Y$ exists such that $M_{i_{\nu}}(Z)=M_{i_{\nu}}(Y)(\nu=1, \cdots, k)$. Hence $M_{i_{1}}, \cdots, M_{i_{k+1}}$ can be realized if and only if $M_{i_{k+1}}=M_{i_{k+1}}(Y)$.

Let us consider the case that $Y$ is not degenerate. Denote by $\left\{d_{n}\right\}$ and $\left\{\epsilon_{n}\right\} \quad(n=1, \cdots$, ad inf.) two sequences of positive numbers such that

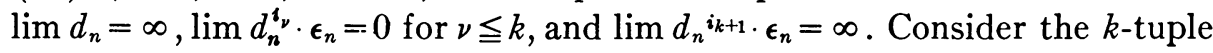
of values

$$
M_{i_{\nu}}(d, \epsilon)=\frac{M_{i_{\nu}}-d^{i \nu} \cdot \epsilon}{1-\epsilon}, \quad \quad \nu=1, \cdots, k .
$$

If $M_{i_{1}}(d, \epsilon), \cdots, M_{i_{k}}(d, \epsilon)$ can be realized as moments of the orders $i_{1}, \cdots, i_{k}$, 
then we shall denote by $Y(d, \epsilon)$ the characteristic chance variable relative to these moments, and by $\bar{Y}(d, \epsilon)$ the arithmetic chance variable defined as follows:

$$
\begin{aligned}
& P[\bar{Y}(d, \epsilon)=d]=P[Y(d, \epsilon)=d](1-\epsilon)+\epsilon, \\
& P[\bar{Y}(d, \epsilon)=x]=P[Y(d, \epsilon)=x](1-\epsilon),
\end{aligned}
$$

for $x \neq d$.

It is obvious that

$$
M_{i_{\nu}}[\bar{Y}(d, \epsilon)]=M_{i_{\nu}}, \quad \quad \nu=1, \cdots, k .
$$

Since $\lim \epsilon_{n}=\lim d_{n}{ }^{i_{\nu}} \epsilon_{n}=0(\nu=1, \cdots, k)$, from Propositions 14 and 8 it follows easily that for almost every $n, Y\left(d_{n}, \epsilon\right)$ exists and is not degenerate for any nonnegative value $\epsilon \leqq \epsilon_{n}$. On account of Proposition 12, $Y\left(d_{n}, \epsilon\right)$ is a continuous function of $\epsilon$ in the interval $\left[0, \epsilon_{n}\right]$. Since $Y\left(d_{n}, \epsilon\right)$ is not degenerate for $0 \leqq \epsilon \leqq \epsilon_{n}$, also $M_{r}\left[Y\left(d_{n}, \epsilon\right)\right]$ is a continuous function of $\epsilon$ for any positive integer $r$. From this it follows that also $M_{r}\left[\bar{Y}\left(d_{n}, \epsilon\right)\right]$ is a continuous function of $\epsilon$ in the interval $\left[0, \epsilon_{n}\right]$. Since

$$
M_{i_{\nu}}\left[\bar{Y}\left(d_{n}, \epsilon\right)\right]=M_{i_{\nu}} \quad(\nu=1, \cdots, k), \quad M_{i_{k+1}}\left[\bar{Y}\left(d_{n}, 0\right)\right]=M_{i_{k+1}}(Y),
$$

we get that $M_{i_{1}}, \cdots, M_{i_{k+1}}$ can be realized as moments if

$$
M_{i_{k+1}}(Y) \leqq M_{i_{k+1}} \leqq M_{i_{k+1}}\left[\bar{Y}\left(d_{n}, \epsilon_{n}\right)\right] .
$$

Because $\lim d_{n}^{i_{k+1} \epsilon_{n}=\infty}$ we obtain easily that $\lim M_{i_{k+1}}\left[\bar{Y}\left(d_{n}, \epsilon_{n}\right)\right]=\infty$ and therefore $M_{i_{1}}, \cdots, M_{i_{k+1}}$ can be realized as moments if $M_{i_{k+1}} \geqq M_{i_{k+1}}(Y)$. From Proposition 8 it follows that this condition is also necessary. Hence we have proved

PROPOSITION 19. Denote by $M_{i_{1}}, \cdots, M_{i_{k}}$ k numbers which can be realized as moments of the orders $i_{1}<i_{2}<\cdots<i_{k}$. Denote by $i_{k+1}$ an integer greater than $i_{k}$ and by $M_{i_{k+1}}$ a certain number. If the chance variable $Y$ which is characteristic relative to $M_{i_{1}}, \cdots, M_{i_{k}}$ is degenerate, then $M_{i_{1}}, \cdots, M_{i_{k+1}}$ can be realized as moments of the orders $i_{1}, \cdots, i_{k+1}$ if and only if $M_{i_{k+1}}=M_{i_{k+1}}(Y)$. If $Y$ is not degenerate, then $M_{i_{1}}, \cdots, M_{i_{k+1}}$ can be realized as moments if and only if $M_{i_{k+1}} \geqq M_{i_{k+1}}(Y)$.

If $M_{i_{k+1}}=M_{i_{k+1}}(Y)$, the characteristic chance variable relative to $M_{i_{1}}, \cdots, M_{i_{k+1}}$ is obviously equal to $Y$ and therefore is degenerate. Since $M_{i_{1}}$ can be realized as a moment of the order $i_{1}$ if and only if $M_{i_{1}} \geqq 0$, we get from Proposition 19

TheOREM 2. Denote by $i_{1}<i_{2}<\cdots<i_{k}$ positive integers and by $M_{i_{1}}, \cdots, M_{i_{k}}$ some numbers. The values $M_{i_{1}}, \cdots, M_{i_{k}}$ can be realized as moments of the orders $i_{1}, \cdots, i_{k}$ if and only if 


$$
M_{i_{1}} \geqq 0, M_{i_{2}} \geqq M_{i_{2}}\left(X_{1}\right), \cdots, M_{i_{k}} \geqq M_{i_{k}}\left(X_{k-1}\right),
$$

where $X_{r}$ denotes the characteristic chance variable relative to $M_{i_{1}}, \cdots, M_{i_{r}}$; if in one of the above relations the equality sign holds, then in all subsequent relations the equality sign must hold.

This theorem gives the solution of Problem 2, since $M_{i_{r}}\left(X_{r-1}\right)$ is a function of $M_{i_{1}}, \cdots, M_{i_{r-1}}$ which can be calculated.

6. Some applications of Theorems 1 and 2. Let us calculate by means of Theorem 2 the inequalities which must be satisfied by the numbers $M_{r}, M_{s}$, $M_{t}$ if they can be realized as moments of the orders $r, s, t$, where $r<s<t$.

According to Theorem 2 the necessary and sufficient conditions are given by

$$
M_{r} \geqq 0, \quad M_{s} \geqq M_{s}\left(X_{1}\right), \quad M_{t} \geqq M_{t}\left(X_{2}\right),
$$

where $X_{1}$ denotes the characteristic chance variable relative to $M_{r}$, and $X_{2}$ denotes the characteristic chance variable relative to $M_{r}$ and $M_{s}$. The degree of $X_{1}$ is less than or equal to 1 . Hence there exists only a single point $a$ with positive probability and therefore $M_{r}=M_{r}\left(X_{1}\right)=a^{r}$. Hence $a=M_{r}^{1 / r}$. It is obvious that

$$
M_{s}\left(X_{1}\right)=a^{s}=M_{r}^{s / r} .
$$

Let us now calculate the chance variable $X_{2}$. The degree of $X_{2}$ is less than or equal to $3 / 2$. Hence only the origin and a single positive value $b$ can have positive probability. The value of $b$ and the probability $P\left(X_{2}=b\right)$ are determined by the equations

$$
M_{r}\left(X_{2}\right)=b^{r} P\left(X_{2}=b\right)=M_{r} ; \quad M_{s}\left(X_{2}\right)=b^{s} P\left(X_{2}=s\right)=M_{s} .
$$

From these equations we obtain

Hence

$$
P\left(X_{2}=b\right)=\frac{M_{r}}{\left(M_{s} / M_{r}\right)^{r /(8-r)}}, \quad b=\left(\frac{M_{s}}{M_{r}}\right)^{1 /(8-r)}
$$

$$
M_{t}\left(X_{2}\right)=b^{t} P\left(X_{2}=b\right)=M_{r}\left(\frac{M_{s}}{M_{r}}\right)^{(t-r) /(s-r)}
$$

From (10), (11), and (12) we get

$$
M_{r} \geqq 0, \quad M_{s} \geqq M_{r}^{s / r}, \quad M_{t} \geqq M_{r}\left(\frac{M_{s}}{M_{r}}\right)^{(t-r) /(s-r)}
$$

If in one of the relations (13) the equality sign holds, then in all subsequent relations the equality sign must hold. These relations are necessary and suffi- 
cient in order that $M_{r}, M_{s}, M_{t}$ can be realized as moments of the orders $r, s, t$.

As an application of Theorem 1 let us calculate the sharp lower limit $a_{d}$ and the sharp upper limit $b_{d}$ if two moments $M_{r}$ and $M_{s}$ are given, where $r<s$. According to the relations (13) we have

$$
M_{r} \geqq 0, \quad M_{s} \geqq M_{r}^{s / r} .
$$

If $M_{r}=0$ (and therefore also $M_{s}=0$ ), or if $M_{r}>0$ and $M_{s}=M_{r}^{s / r}$, the chance variable $X$ which is characteristic relative to $M_{r}$ and $M_{s}$ is degenerate and we have $a_{d}=b_{d}=P(X<d)$. Since $P(X<x)=0$ for $x \neq M_{r}^{1 / r}$ and $P\left(X=M_{r}^{1 / r}\right)=1$, we have

$$
\begin{array}{ll}
a_{d}=b_{d}=1, & \text { for } d>M_{r}^{1 / r}, \\
a_{d}=b_{d}=0, & \text { for } d \leqq M_{r}^{1 / r} .
\end{array}
$$

Now we have to consider the case that

$$
M_{r}>0, \quad M_{s}>M_{r}^{s / r} .
$$

In order to calculate $\lambda_{d}$ we have to consider the expressions:

$$
M_{r}(d, \lambda)=\frac{M_{r}-d^{r} \lambda}{1-\lambda}, \quad M_{s}(d, \lambda)=\frac{M_{s}-d^{s} \lambda}{1-\lambda} .
$$

From Theorem 2 it follows that for any $\lambda$ for which $M_{r}(d, \lambda)>0$ and $M_{s}(d, \lambda)>\left[M_{r}(d, \lambda)\right]^{s / r}, M_{r}(d, \lambda)$ and $M_{s}(d, \lambda)$ can be realized as moments of the orders $r, s$, and the corresponding characteristic chance variable is not degenerate. Hence either $M_{r}\left(d, \lambda_{d}\right)=0$ or $M_{s}\left(d, \lambda_{d}\right)=\left[M_{r}\left(d, \lambda_{d}\right)\right]^{s / r}$ must hold. That is to say, $\lambda_{d}$ is either equal to $M_{r} / d^{r}$ or is the root of the equation

$$
\frac{M_{s}-d^{s} \lambda}{1-\lambda}=\left[\frac{M_{r}-d^{r} \lambda}{1-\lambda}\right]^{s / r} \text {. }
$$

We have $\lambda_{d}=M_{r} / d^{r}$ if and only if the smallest positive root of (15) is greater than or equal to $M_{r} / d^{r}$. It is easy to show that this is the case if $M_{r} / d^{r}$ $\leqq M_{s} / d^{s}$. Hence we have:

If $M_{r} / d^{r} \leqq M_{s} / d^{s}$ then $\lambda_{d}=M_{r} / d^{r}$, and if $M_{r} / d^{r}>M_{s} / d^{s}$ then $\lambda_{d}$ is equal to the smallest positive root of (15).

If $\lambda_{d}=M_{r} / d^{r}$ then the chance variable $Y_{d}$ which is characteristic relative to $M_{r}\left(d, \lambda_{d}\right)$ is given as follows: $P\left(Y_{d}=0\right)=1$ and $P\left(Y_{d}=x\right)=0$ for $x \neq 0$. Hence the chance variable $\bar{Y}_{d}$ is given as follows:

$$
\begin{aligned}
& P\left(\bar{Y}_{d}=0\right)=1-\lambda_{d}=1-M_{r} / d^{r}, \\
& P\left(\bar{Y}_{d}=d\right)=\lambda_{d}=M_{r} / d^{r} .
\end{aligned}
$$




\section{Hence}

(16) $a_{d}=P\left(\bar{Y}_{d}<d\right)=1-M_{r} / d^{r} ; \quad b_{d}=P\left(\bar{Y}_{d} \leqq d\right)=1, \quad M_{r} / d^{r} \leqq M_{s} / d^{s}$.

Let us now consider the case that $M_{r} / d^{r}>M_{s} / d^{s}$. Then $\lambda_{d}$ is the smallest positive root of (15). The chance variable $Y_{d}$ which is characteristic relative to $M_{r}\left(d, y_{d}\right)$ is given as follows: $P\left(Y_{d}=\delta\right)=1$ where

$$
\delta^{r}=\frac{M_{r}-d^{r} \cdot \lambda_{d}}{1-\lambda_{d}}, \quad \text { or } \quad \frac{\delta^{r}}{d^{r}}=\frac{M_{r} / d^{r}-\lambda_{d}}{1-\lambda_{d}} .
$$

The chance variable $\bar{Y}_{d}$ is given as follows:

$$
P\left(\bar{Y}_{d}=\delta\right)=P\left(Y_{d}=\delta\right) \cdot\left(1-\lambda_{d}\right)=\left(1-\lambda_{d}\right), \quad P\left(\bar{Y}_{d}=d\right)=\lambda_{d} .
$$

We shall show that $\delta<d$. One can easily see that $M_{r} / d^{r}<1$. In fact, if $M_{r} / d^{r}>1$, then

$$
\left(\frac{M_{r}}{d^{r}}\right)^{s / r}>\frac{M_{r}}{d^{r}}>\frac{M_{s}}{d^{s}}
$$

and therefore $M_{r}{ }_{r} / r>M_{s}$ which is not possible. The inequality $\delta / d<1$ follows from (17) on account of $M_{r} / d^{r}<1$. Hence we have

$$
\begin{aligned}
& a_{d}=P\left(\bar{Y}_{d}<d\right)=P\left(\bar{Y}_{d}=\delta\right)=1-\lambda_{d} ; \quad b_{d}=P\left(\bar{Y}_{d} \leqq d\right)=1, \\
& M_{r} / d^{r}>M_{s} / d^{s} .
\end{aligned}
$$

The equations (16) and (18) give the complete formulas for $a_{d}$ and $b_{d}$ if two moments $M_{r}$ and $M_{s}$ are given.

If $s=2 r$ the root $\lambda_{d}$ of (15) is given by the expression:

Hence we get

$$
\lambda_{d}=\frac{M_{2 r}-M_{r}^{2}}{\left(d^{r}-M_{r}\right)^{2}+\left(M_{2 r}-M_{r}^{2}\right)} .
$$

$$
\begin{aligned}
& a_{d}=1-\lambda_{d}=1-\frac{M_{2 r}-M_{r}^{2}}{\left(d^{r}-M_{r}\right)^{2}+\left(M_{2 r}-M_{r}^{2}\right)}, \\
& b_{d}=1,
\end{aligned}
$$$$
M_{r} / d^{r}>M_{s} / d^{s},
$$

The sharp lower limits given in the formulas (16) and (19) are identical with the lower limits in the formulas (3) given by Cantelli.

COLUMBia University,

NEW YoRK, N. Y. 